\title{
Calculation of Gas Dynamics and Radiation of High-Altitude Jets
}

\author{
A.M. Molchanov, V.E.Popov \\ Moscow Aviation Institute, Moscow, 125993, Russian Federation \\ alexmol_2000@mail.ru
}

\begin{abstract}
A method for calculating high-altitude jets exhausting into a rarefied gas has been developed. This method is based on the use of quasi-gas-dynamic (QGD) equations. The solution of quasigasdynamic equations requires the use of significantly smaller computer resources as compared with the methods of direct numerical simulation. The equations of the continuity of gas mixture components and the equations for vibrational energies transfer in the quasi-gas-dynamic formulation were obtained from the Boltzmann equation. It was shown that when calculating with the use of QGD, the radiation intensity is significantly lower than when calculating the standard system of Navier-Stokes equations (NS). This is due to the decrease in temperature (translational, rotational and vibrational) in the mixing layer.
\end{abstract}

Keywords: rarefied gas, quasi-gas-dynamic equations, high-altitude jets.

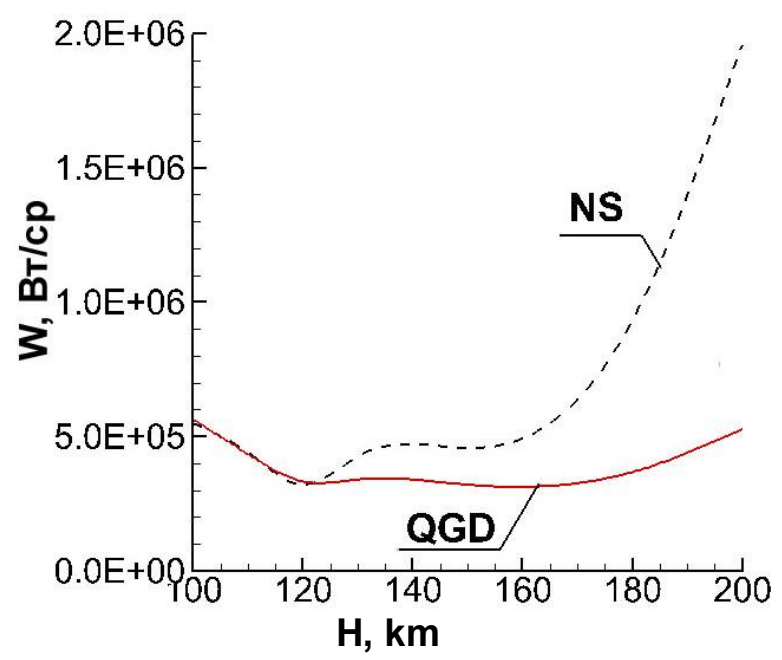

Integrated radiation (range $1 \div 7 \mu \mathrm{m}$ ) variation with height:

NS is calculation using the Navier - Stokes equations taking into account thermal nonequilibrium; QGD is calculation using quasi-gas-dynamic equations taking into account thermal nonequilibrium

$$
\begin{aligned}
\frac{\partial}{\partial t}\left(\mathrm{E}_{V, m}\right)+\frac{\partial}{\partial x_{i}}\left(\mathrm{E}_{V, m} u_{i}\right) & =\frac{\partial}{\partial x_{i}}\left[\tau \frac{\partial}{\partial x_{j}}\left(u_{j} u_{i} \mathrm{E}_{V, m}\right)\right]+\frac{\partial}{\partial x_{i}}\left(\tau \frac{\mathrm{E}_{V, m}}{\rho} \frac{\partial p}{\partial x_{i}}\right)+ \\
& +\frac{\partial}{\partial x_{i}}\left[\frac{1}{\operatorname{Pr}} \tau p \frac{\partial}{\partial x_{i}}\left(\frac{\mathrm{E}_{V, m}}{\rho}\right)\right]+\dot{\mathrm{E}}_{V, m}
\end{aligned}
$$




\title{
Расчет газовой динамики и излучения высотных струй
}

\author{
А.М. Молчанов, В.Э. Попов \\ Московский авиаџионный институт (Национальный исследовательский университет), \\ Российская Федерация, Москва, 125993 \\ alexmol_2000@mail.ru
}

\begin{abstract}
Аннотация
Разработан метод расчета высотных струй, истекающих в разреженный газ. Метод основан на использовании квазигазодинамических (КГД) уравнений. Расчет этим методом требует использования существенно меньших компьютерных ресурсов по сравнению с методами прямого численного моделирования. Из уравнения Больцмана получены уравнения неразрывности компонентов газовой смеси и уравнения переноса колебательных энергий в квазигазодинамической постановке. Показано, что при расчете с использованием КГД уравнений интенсивность излучения существенно ниже, чем при расчете на основе стандартной системы уравнений Навье-Стокса (НС). Это обусловлено снижением температур (поступательной, вращательной и колебательных) в слое смешения.
\end{abstract}

Ключевые слова: разреженный газ, квазигазодинамические уравнения, высотные струи.

\section{1. Введение}

Расчет высотных струй представляет серьезную проблему. В основном, это связано с нарушением условия сплошности разреженной среды и ярко выраженной неравновесностью между различными энергетическими модами.

Основным параметром, характеризующим степень разреженности течения, является число Кнудсена

$$
\mathrm{Kn}=\frac{l}{L},
$$

где $l$ - средняя длина свободного пробега молекул; $L$ - характерный линейный размер рассматриваемой области течения. Обычно газ считается сплошным, если $\mathrm{Kn}<0.01$. Условие $\mathrm{Kn}>10$ характерно для свободно-молекулярных течений, когда столкновения между частицами практически отсутствуют.

При промежуточных числах $(0.01<\mathrm{Kn}<10)$ газ считается разреженным. Расчет таких течений представляет большую сложность, т.к. с одной стороны, уравнения Навье-Стокса $(\mathrm{HC})$, полученные в приближении $\mathrm{Kn} \rightarrow 0$, теряют свою точность при анализе указанных режимов; с другой стороны, расчет таких течений методами кинетической теории требует неоправданно больших вычислительных ресурсов, что обусловлено высокой плотностью газа.

Численный анализ таких течений может проводиться на основе методов прямого численного моделирования - методов Монте-Карло, или DSMC методов $[1,2,3]$. Однако расчет этими методами также требует использования очень больших компьютерных ресурсов.

Поэтому для течений в диапазоне чисел Кнудсена $0.01 \div 10$ желательно применение более простых моделей.

В Институте прикладной математики разработана модель, получившая название квазигазодинамические (КГД) уравнения [4,5]. 
Этот подход основан на использовании математической модели, обобщающей систему уравнений Навье - Стокса и отличающейся от нее дополнительными диссипативными слагаемыми с малым параметром в качестве коэффициента. Принципиальным и существенным отличием КГД подхода от теории Навье-Стокса явилось использование процедуры пространственно-временного осреднения для определения основных газодинамических величин - плотности, скорости, температуры и т.п. Авторы $[4,5]$ указывают область применимости модели до $\mathrm{Kn}=10$.

\section{2. Квазигазодинамические уравнения}

Основные уравнения сохранения - квазигазодинамические уравнения, записанные для разреженного однородного совершенного газа с постоянной теплоемкостью, имеют следующий вид [5]:

$$
\begin{gathered}
\frac{\partial \rho}{\partial t}+\frac{\partial}{\partial x_{i}}\left(\rho u_{i}\right)=\frac{\partial}{\partial x_{i}}\left\{\tau\left[\frac{\partial}{\partial x_{j}}\left(\rho u_{j} u_{i}\right)+\frac{\partial p}{\partial x_{i}}\right]\right\} \\
\frac{\partial}{\partial t}\left(\rho u_{j}\right)+\frac{\partial}{\partial x_{i}}\left(\rho u_{i} u_{j}+p \delta_{i j}\right)=\frac{\partial}{\partial x_{i}}\left\{\tau\left[\frac{\partial}{\partial x_{k}}\left(\rho u_{k} u_{i} u_{j}\right)+\frac{\partial}{\partial x_{i}}\left(u_{j} p\right)+\frac{\partial}{\partial x_{j}}\left(u_{i} p\right)\right]\right\}+\frac{\partial}{\partial x_{j}}\left[\tau \frac{\partial}{\partial x_{i}}\left(u_{i} p\right)\right] \\
\frac{\partial}{\partial t}(\rho E)+\frac{\partial}{\partial x_{i}}\left[\rho u_{i}\left(E+\frac{p}{\rho}\right)\right]= \\
=\frac{\partial}{\partial x_{i}}\left\{\tau \frac{\partial}{\partial x_{j}}\left[u_{j} u_{i}(\rho E+2 p)\right]\right\}+\frac{\partial}{\partial x_{i}}\left[\tau \frac{\partial}{\partial x_{i}}\left(\frac{1}{2} u_{k}^{2} p\right)\right]+ \\
+\frac{\partial}{\partial x_{i}}\left(\tau \frac{\gamma}{(\gamma-1)} \frac{p}{\rho} \frac{\partial p}{\partial x_{i}}\right)+\frac{\partial}{\partial x_{i}}\left[\frac{1}{\operatorname{Pr}} \frac{\gamma}{(\gamma-1)} \tau p \frac{\partial}{\partial x_{i}}\left(\frac{p}{\rho}\right)\right]
\end{gathered}
$$

Здесь используются следующие обозначения: $\rho$ - плотность газа; $u_{j}$ - компонента скорости в $j$-м направлении; $p$ - давление; $\gamma$ - показатель адиабаты; $\operatorname{Pr}$ - число Прандтля; $E$ - полная энергия

$$
E=e+\frac{1}{2} u_{k}^{2}
$$

где $e-$ удельная внутренняя энергия.

Система (2)-(4) замыкается уравнениями состояния

$$
p=\rho R T, \quad e=c_{v} T=\frac{1}{\gamma-1} \frac{p}{\rho}
$$

и формулой для $\tau$

$$
\tau=\mu / p
$$

где $\mu$ - коэффициент динамической вязкости; $\tau$ - релаксационный параметр, имеющий смысл максвелловского времени релаксации; $T$ - поступательная температура.

\section{3. Квазигазодинамические уравнения неразрывности компонентов газовой смеси}

Уравнение неразрывности для отдельного компонента $s$ газовой смеси выводится аналогично общему уравнению неразрывности (2). В одножидкостном приближении [5] оно имеет вид

$$
\frac{\partial \rho_{s}}{\partial t}+\frac{\partial}{\partial x_{i}}\left(\rho_{s} u_{i}\right)=\frac{\partial g_{s, i}}{\partial x_{i}}+\dot{w}_{s}, \quad s=1,2, \ldots, N_{C}
$$


где $\rho_{s}$ - массовая (парциальная) плотность компонента $s ; \dot{w}_{s}-$ скорость образования компонента $s$ в результате химических реакций; $N_{C}$ - число компонентов газовой смеси.

Поток $g_{s, i}$ определяется формулой

$$
\begin{aligned}
g_{s, i} & =\tau\left[\frac{\partial}{\partial x_{j}}\left(\rho_{s} u_{j} u_{i}\right)+\frac{\partial p_{s}}{\partial x_{i}}\right]=\tau\left[\frac{\partial}{\partial x_{j}}\left(\rho_{s} u_{j} u_{i}\right)+\frac{\partial}{\partial x_{i}}\left(p X_{s}\right)\right] \\
& =\tau\left[\frac{\partial}{\partial x_{j}}\left(\rho C_{s} u_{j} u_{i}\right)+\frac{\partial}{\partial x_{i}}\left(p C_{s}\right)+\frac{\partial}{\partial x_{i}}\left[p\left(X_{s}-C_{s}\right)\right]\right]
\end{aligned}
$$

где $X_{s}-$ мольная (молярная) доля компонента $s ; C_{s}$ - массовая доля компонента $s$

$$
C_{s}=\rho_{s} / \rho
$$

Пренебрегая последним членом в этом выражении и обобщая уравнение на случай произвольного числа Шмидта $\mathrm{Sc}$, получаем следующую формулу для потока $g_{s, i}$

$$
g_{s, i}=\tau\left[\frac{\partial}{\partial x_{j}}\left(\rho C_{s} u_{j} u_{i}\right)+C_{s} \frac{\partial p}{\partial x_{i}}+\frac{1}{\mathrm{Sc}} p \frac{\partial C_{s}}{\partial x_{i}}\right]
$$

Указанные допущения аналогичны предположению, часто используемому в плотных течениях, о том, что диффузионный поток в многокомпонентных смесях определяется по закону Фика с использованием обобщенного числа Шмидта, которое одинаково для всех компонентов смеси.

Заметим, что при суммировании уравнений (8) по всем компонентам и при использовании формулы (9) для потоков, мы получаем общее уравнение неразрывности в форме (2).

Если ввести обозначение

$$
g_{i}=\tau\left[\frac{\partial}{\partial x_{j}}\left(\rho u_{j} u_{i}\right)+\frac{\partial p}{\partial x_{i}}\right]=\tau \frac{\partial}{\partial x_{j}}\left(\rho u_{j} u_{i}+\delta_{j i} p\right),
$$

то общее уравнение неразрывности (2) для газовой смеси запишется в виде

$$
\frac{\partial \rho}{\partial t}+\frac{\partial}{\partial x_{i}}\left(\rho u_{i}\right)=\frac{\partial g_{i}}{\partial x_{i}},
$$

а поток $g_{s, i}-$

$$
g_{s, i}=C_{s} g_{i}+\tau\left(\rho u_{j} u_{i}+\frac{1}{\mathrm{Sc}} \delta_{i j} p\right) \frac{\partial C_{s}}{\partial x_{j}}
$$

\section{4. Квазигазодинамические уравнения энергий для колебательно неравновесного газа}

Внутренняя энергия каждого компонента $s$ складывается из поступательной $e_{T, s}$, вращательной $e_{R, s}$, колебательной $e_{V, m}$ и химической $h_{s}^{0}$ составляющих, и их вклад во внутреннюю энергию газовой смеси пропорционален массовой доли:

$$
e=\sum_{s=1}^{N c} C_{s} e_{T, s}+\sum_{s=1}^{N c} C_{s} e_{R, s}+\sum_{m=1}^{N_{M}} C_{s(m)} e_{V, m}+\sum_{s=1}^{N c} C_{s} h_{s}^{0},
$$

где $C_{s(m)}$ - массовая доля компонента $s$, к которому относится $m$-я колебательная мода; $N_{M}$ - количество колебательных мод. 
При использовании модели гармонического осциллятора среднее число $m$-х колебательных квантов $\alpha_{m}$, приходящихся на одну молекулу, определяется формулой

$$
\alpha_{m}=r_{m} \frac{1}{\exp \left(\theta_{m} / T_{V, m}\right)-1},
$$

где $\theta_{m}$ - характеристическая колебательная температура $m$-й колебательной моды; $T_{V, m}-$ соответствующая колебательная температура; $r_{m}$ - кратность вырождения $m$-й моды молекулы.

Энергия $m$-й колебательной моды определяется соотношением $h v_{m}=k \theta_{m}$.

Таким образом, энергия молекулы, запасенная в $m$-м типе колебаний, равна

$$
\varepsilon_{V, m}=k \theta_{m} \alpha_{m}=\frac{r_{m} k \theta_{m}}{\exp \left(\theta_{m} / T_{V, m}\right)-1}
$$

Для того, чтобы получить выражение для энергии, приходящейся на 1 моль вещества, необходимо умножить соответствующую энергию молекулы на число Авогадро $N_{A}$. Для удельной энергии (на единицу массы вещества) надо затем разделить полученное выражение на молярную массу М

$$
e_{V, m}=\frac{r_{m} R \theta_{m}}{\exp \left(\theta_{m} / T_{V, m}\right)-1}, \quad m=1,2, \ldots, N_{M}
$$

Здесь использовались соотношения

$$
k N_{A}=R_{U}, \quad R=R_{U} / \mathrm{M},
$$

где $R_{U}$ - универсальная газовая постоянная; $R$ - газовая постоянная данного вещества.

Получим уравнение для $\mathrm{E}_{V, m}$ - колебательной энергии $m$-й колебательной моды, содержащейся в единице объема газа.

Определим функцию распределения для одинаковых молекул через выражение

$$
f(t, \mathbf{x}, \boldsymbol{\xi}) \mathrm{d} \mathbf{x} \mathrm{d} \xi=m_{0} \mathrm{~d} N,
$$

где $\mathrm{d} N$ - ожидаемое число молекул в элементе пространства $\mathrm{d} \mathbf{x}$ в окрестности точки $\mathbf{x}$, обладающих скоростями в элементе пространства скоростей $\mathrm{d} \xi$, в окрестности точки $\xi$ в момент времени $t ; m_{0}$ - масса частицы.

Поведение функции распределения $f$ описывается уравнением Больцмана [5]

$$
\frac{\partial f}{\partial t}+\xi_{i} \frac{\partial f}{\partial x_{i}}=\mathrm{J}(f, f),
$$

где $\mathrm{J}(f, f)$ - интеграл столкновений, который является нелинейным функционалом и определяет изменение функции распределения в результате парных столкновений.

Для построения моментных уравнений, описывающих течения вязкого газа, традиционно используются приближения для функции $f$ в виде разложения по малому параметру вблизи равновесного значения с последующим осреднением полученного кинетического уравнения с сумматорными инвариантами.

Для построения квазигазодинамических уравнений, учитывающих поступательно-колебательную неравновесность, заменим функцию распределения $f$ ее приближенным значением $f^{\mathrm{QGD}}$, которое представляет собой разложение по малому параметру в окрестности равновесной функции распределения $f^{0}$ следующего вида [5]: 


$$
f^{\mathrm{QGD}}=f^{0}-\tau \xi_{i} \frac{\partial f^{0}}{\partial x_{i}}
$$

Формальная замена $f \rightarrow f^{\mathrm{QGD}}$ в конвективном слагаемом уравнения Больцмана (17) приводит к приближенному уравнению

$$
\frac{\partial f}{\partial t}+\xi_{i} \frac{\partial f^{0}}{\partial x_{i}}-\xi_{i} \frac{\partial}{\partial x_{i}}\left(\tau \xi_{j} \frac{\partial f^{0}}{\partial x_{j}}\right)=\mathrm{J}(f, f)
$$

Чтобы получить уравнение для колебательной энергии $\mathrm{E}_{V, m}$, необходимо осреднить (18) с весом $\varepsilon_{V, m}$, определяемым по формуле (15).

Учитывая, что

$$
\mathrm{E}_{V, m}=\int \varepsilon_{V, m} f \mathrm{~d} \xi=\int \varepsilon_{V, m} f^{0} \mathrm{~d} \xi,
$$

получим следующее уравнение

$$
\frac{\partial}{\partial t}\left(\mathrm{E}_{V, m}\right)+\frac{\partial}{\partial x_{i}}\left(\mathrm{E}_{V, m} u_{i}\right)=\frac{\partial}{\partial x_{i}}\left\{\tau \frac{\partial}{\partial x_{j}}\left[\left(\rho u_{j} u_{i}+\delta_{i j} p\right) \frac{\mathrm{E}_{V, m}}{\rho}\right]\right\}+\dot{\mathrm{E}}_{V, m},
$$

где обменный член определяется формулой

$$
\dot{\mathrm{E}}_{V, m}=\int \mathrm{J}(f, f) \varepsilon_{V, m} f \mathrm{~d} \xi
$$

Первый член в правой части уравнения (20) можно представить в виде

$$
\frac{\partial}{\partial x_{i}}\left\{\tau \frac{\partial}{\partial x_{j}}\left[\left(\rho u_{j} u_{i}+\delta_{i j} p\right) \frac{\mathrm{E}_{V, m}}{\rho}\right]\right\}=\frac{\partial}{\partial x_{i}}\left[\tau \frac{\partial}{\partial x_{j}}\left(u_{j} u_{i} \mathrm{E}_{V, m}\right)\right]+\frac{\partial}{\partial x_{i}}\left(\tau \frac{\mathrm{E}_{V, m}}{\rho} \frac{\partial p}{\partial x_{i}}\right)+\frac{\partial}{\partial x_{i}}\left[\tau p \frac{\partial}{\partial x_{i}}\left(\frac{\mathrm{E}_{V, m}}{\rho}\right)\right]
$$

Изложенный здесь способ получения моментных уравнений приводит к выражениям для теплового потока с числом Прандтля, равным единице. Для обобщения уравнений на случай произвольного числа Прандтля последнее слагаемое в (21) следует домножить на величину $\operatorname{Pr}^{-1}$.

Окончательно получаем уравнение для колебательной энергии

$$
\frac{\partial}{\partial t}\left(\mathrm{E}_{V, m}\right)+\frac{\partial}{\partial x_{i}}\left(\mathrm{E}_{V, m} u_{i}\right)=\frac{\partial}{\partial x_{i}}\left[\tau \frac{\partial}{\partial x_{j}}\left(u_{j} u_{i} \mathrm{E}_{V, m}\right)\right]+\frac{\partial}{\partial x_{i}}\left(\tau \frac{\mathrm{E}_{V, m}}{\rho} \frac{\partial p}{\partial x_{i}}\right)+\frac{\partial}{\partial x_{i}}\left[\frac{1}{\operatorname{Pr}} \tau p \frac{\partial}{\partial x_{i}}\left(\frac{\mathrm{E}_{V, m}}{\rho}\right)\right]+\dot{\mathrm{E}}_{V, m}
$$

С учетом (10) это уравнение примет вид

$$
\begin{gathered}
\frac{\partial}{\partial t}\left(\mathrm{E}_{V, m}\right)+\frac{\partial}{\partial x_{i}}\left(\mathrm{E}_{V, m} u_{i}\right)=\frac{\partial F_{m, i}}{\partial x_{i}}+\dot{\mathrm{E}}_{V, m}, \\
\text { где } \quad F_{m, i}=\frac{\mathrm{E}_{V, m}}{\rho} g_{i}+\tau\left(\rho u_{j} u_{i}+\frac{1}{\operatorname{Pr}} \delta_{j i} p\right) \frac{\partial}{\partial x_{j}}\left(\frac{\mathrm{E}_{V, m}}{\rho}\right)
\end{gathered}
$$

Рассмотрим теперь поступательную и вращательную части полной энергии.

Предполагая равновесие между поступательной и вращательной энергиями, можно получить уравнение для энергии 


$$
E_{T R}=e_{T R}+\frac{1}{2} u_{k}^{2}
$$

где $e_{T R}$ - поступательно-вращательная часть внутренней энергии. Соответствующий показатель адиабаты определяется по формуле

$$
\gamma_{T R}=\frac{5+i_{R}}{3+i_{R}}
$$

где $i_{R}$ - число вращательных степеней свободы молекулы: $i_{R}=0$ для одноатомных молекул, $i_{R}=2$ для линейных молекул, $i_{R}=3$ для многоатомных нелинейных молекул (число атомов больше 2).

Уравнение для $E_{T R}$ совпадает по форме с уравнением (4)

$$
\begin{aligned}
\frac{\partial}{\partial t}\left(\rho E_{T R}\right)+\frac{\partial}{\partial x_{i}}\left[\rho u_{i}\left(E_{T R}+\frac{p}{\rho}\right)\right] & =\frac{\partial}{\partial x_{i}}\left\{\tau \frac{\partial}{\partial x_{j}}\left[u_{j} u_{i}\left(\rho E_{T R}+2 p\right)\right]\right\}+\frac{\partial}{\partial x_{i}}\left[\tau \frac{\partial}{\partial x_{i}}\left(\frac{1}{2} u_{k}^{2} p\right)\right]+ \\
& +\frac{\partial}{\partial x_{i}}\left(\tau h_{T R} \frac{\partial p}{\partial x_{i}}\right)+\frac{\partial}{\partial x_{i}}\left(\frac{1}{\operatorname{Pr}} \tau p \frac{\partial h_{T R}}{\partial x_{i}}\right)+S_{T R},
\end{aligned}
$$

где $h_{T R}=\frac{\gamma_{T R}}{\left(\gamma_{T R}-1\right)} R T-$ поступательно-вращательная энтальпия; $S_{T R}-$ обменный член, которой уже не равен нулю, т.к. имеется обмен между поступательно-вращательной и колебательными энергиями.

Складывая уравнения (25) и (22) для всех колебательных мод, получаем уравнение полной энергии для колебательно неравновесного однородного газа

$$
\begin{aligned}
\frac{\partial}{\partial t}\left(\rho E_{T R V}\right)+\frac{\partial}{\partial x_{i}}\left[\rho u_{i}\left(E_{T R V}+\frac{p}{\rho}\right)\right] & =\frac{\partial}{\partial x_{i}}\left\{\tau \frac{\partial}{\partial x_{j}}\left[\rho u_{j} u_{i}\left(E_{T R V}+2 \frac{p}{\rho}\right)\right]\right\}+\frac{\partial}{\partial x_{i}}\left[\tau \frac{\partial}{\partial x_{i}}\left(\frac{1}{2} u_{k}^{2} p\right)\right]+ \\
& +\frac{\partial}{\partial x_{i}}\left(\tau h_{T R V} \frac{\partial p}{\partial x_{i}}\right)+\frac{\partial}{\partial x_{i}}\left[\frac{1}{\operatorname{Pr}} \tau p \frac{\partial h_{T R V}}{\partial x_{i}}\right] \\
E_{T R V}=E_{T R} & +\frac{1}{\rho} \sum_{m=1}^{N m} \mathrm{E}_{V, m}, \quad h_{T R V}=h_{T R}+\frac{1}{\rho} \sum_{m=1}^{N m} \mathrm{E}_{V, m}
\end{aligned}
$$

Очевидно, что

$$
S_{T R}+\sum_{m=1}^{N m} \dot{\mathrm{E}}_{V, m}=0
$$

Нетрудно показать, что при использовании допущения о подобии диффузионного переноса и теплового потока, т.е. при $\mathrm{Pr}=\mathrm{Sc}$, уравнение энергии в форме (26) может использоваться и для смеси газов.

Окончательно получаем уравнение полной энергии для колебательно неравновесной газовой смеси

$$
\begin{aligned}
& \frac{\partial}{\partial t}(\rho E)+\frac{\partial}{\partial x_{i}}\left(\rho u_{i} H\right)= \\
& \quad=\frac{\partial}{\partial x_{i}}\left\{\tau \frac{\partial}{\partial x_{j}}\left[\rho u_{j} u_{i}\left(H+\frac{p}{\rho}\right)\right]\right\}+\frac{\partial}{\partial x_{i}}\left[\tau \frac{\partial}{\partial x_{i}}\left(\frac{1}{2} u_{k}^{2} p\right)\right]+\frac{\partial}{\partial x_{i}}\left(\tau h \frac{\partial p}{\partial x_{i}}\right)+\frac{\partial}{\partial x_{i}}\left(\frac{1}{\operatorname{Pr}} \tau p \frac{\partial h}{\partial x_{i}}\right)
\end{aligned}
$$


Здесь $H=E+\frac{p}{\rho}-$ полная энтальпия газовой смеси.

\section{5. Вычисление обменных членов в уравнении колебательной энергии}

Обменный член в уравнении (22) $\dot{\mathrm{E}}_{V, m}$ имеет физический смысл возбуждения или дезактивации колебательных степеней свободы молекул при неупругих соударениях. Этот процесс может происходить несколькими путями: 1) путем непосредственного перехода кинетической энергии сталкивающихся молекул в колебательную энергию, и наоборот (процесс прямого возбуждения или дезактивации), обозначаемый как T-V (V-T) переход; 2) путем обмена энергией между колебательными степенями свободы сталкивающихся молекул (процесс колебательно-колебательного обмена), обозначаемый как V-V переход; 3) вследствие спонтанной излучательной дезактивации. V-V переходы бывают как внутримолекулярные, так и межмолекулярные.

В данной работе учитывались следующие энергетические переходы [6]:

\section{V-T nроцессы:}

1. $\mathrm{N}_{2}(1)+\mathrm{M} \rightleftarrows \mathrm{N}_{2}(0)+\mathrm{M}$

2. $\mathrm{CO}_{2}\left(01^{1} 0\right)+\mathrm{M}=\mathrm{CO}_{2}\left(00^{\circ} 0\right)+\mathrm{M}$

3. $\mathrm{CO}(1)+\mathrm{M} \rightleftarrows \mathrm{CO}(0)+\mathrm{M}$

4. $\mathrm{H}_{2} \mathrm{O}(010)+\mathrm{M} \rightleftarrows \mathrm{H}_{2} \mathrm{O}(000)+\mathrm{M}$

5. $\mathrm{H}_{2}(1)+\mathrm{M} \rightleftarrows \mathrm{H}_{2}(0)+\mathrm{M}$

6. $\mathrm{HCl}(1)+\mathrm{M} \rightleftarrows \mathrm{HCl}(0)+\mathrm{M}$

8. $\mathrm{H}_{2} \mathrm{O}(100)+\mathrm{M} \rightleftarrows \mathrm{H}_{2} \mathrm{O}(000)+\mathrm{M}$

9. $\mathrm{H}_{2} \mathrm{O}(001)+\mathrm{M} \rightleftarrows \mathrm{H}_{2} \mathrm{O}(000)+\mathrm{M}$

10. $\mathrm{O}_{2}(1)+\mathrm{M} \rightleftarrows \mathrm{O}_{2}(0)+\mathrm{M}$

11. $\mathrm{OH}(1)+\mathrm{M} \rightleftarrows \mathrm{OH}(0)+\mathrm{M}$

12. $\mathrm{CO}_{2}\left(00^{0} 1\right)+\mathrm{M}=\mathrm{CO}_{2}\left(00^{\circ} 0\right)+\mathrm{M}$

13. $\mathrm{Cl}_{2}(1)+\mathrm{M} \rightleftarrows \mathrm{Cl}_{2}(0)+\mathrm{M}$

7. $\mathrm{NO}(1)+\mathrm{M} \rightleftarrows \mathrm{NO}(0)+\mathrm{M}$

внутримолекулярные V-V процессы:

14. $\mathrm{CO}_{2}\left(00^{0} 1\right)+\mathrm{M}=\left\{\begin{array}{l}\mathrm{CO}_{2}\left(03^{1} 0\right)+\mathrm{M} \\ \mathrm{CO}_{2}\left(11^{1} 0\right)+\mathrm{M}\end{array}\right.$

15. $\mathrm{CO}_{2}\left(10^{0} 0\right)+\mathrm{M}=\mathrm{CO}_{2}\left(02^{0} 0\right)+\mathrm{M}$

16. $\mathrm{H}_{2} \mathrm{O}(100)+\mathrm{M} \rightleftarrows \mathrm{H}_{2} \mathrm{O}(020)+\mathrm{M}$

17. $\mathrm{H}_{2} \mathrm{O}(001)+\mathrm{M} \rightleftarrows \mathrm{H}_{2} \mathrm{O}(020)+\mathrm{M}$

18. $\mathrm{H}_{2} \mathrm{O}(001)+\mathrm{M} \rightleftarrows \mathrm{H}_{2} \mathrm{O}(100)+\mathrm{M}$

\section{межмолекулярные V-V' процессы:}

19. $\mathrm{CO}_{2}\left(00^{0} 1\right)+\mathrm{N}_{2}(0)=\mathrm{CO}_{2}\left(00^{0} 0\right)+\mathrm{N}_{2}(1)$

20. $\mathrm{CO}_{2}\left(00^{0} 1\right)+\mathrm{CO}(0)=\mathrm{CO}_{2}\left(00^{0} 0\right)+\mathrm{CO}(1)$

21. $\mathrm{CO}(1)+\mathrm{N}_{2}(0)=\mathrm{CO}(0)+\mathrm{N}_{2}(1)$

22. $\mathrm{N}_{2}(1)+\mathrm{NO}(0)=\mathrm{N}_{2}(0)+\mathrm{NO}(1)$

23. $\mathrm{CO}(1)+\mathrm{NO}(0)=\mathrm{CO}(0)+\mathrm{NO}(1)$

24. $\mathrm{CO}_{2}\left(00^{0} 1\right)+\mathrm{NO}(0)=\mathrm{CO}_{2}\left(00^{0} 0\right)+\mathrm{NO}(1)$

25. $\mathrm{CO}_{2}\left(00^{0} 0\right)+\mathrm{N}_{2}(1)=\left\{\begin{array}{l}\mathrm{CO}_{2}\left(03^{1} 0\right)+\mathrm{N}_{2}(0) \\ \mathrm{CO}_{2}\left(11^{1} 0\right)+\mathrm{N}_{2}(0)\end{array}\right.$

26. $\mathrm{CO}_{2}\left(00^{0} 0\right)+\mathrm{CO}(1)=\left\{\begin{array}{l}\mathrm{CO}_{2}\left(03^{1} 0\right)+\mathrm{CO}(0) \\ \mathrm{CO}_{2}\left(11^{1} 0\right)+\mathrm{CO}(0)\end{array}\right.$

27. $\mathrm{N}_{2}(1)+\mathrm{O}_{2}(0)=\mathrm{N}_{2}(0)+\mathrm{O}_{2}(1)$

28. $\mathrm{CO}_{2}\left(01^{1} 1\right)+\mathrm{N}_{2}(0)=\mathrm{CO}_{2}\left(01^{1} 0\right)+\mathrm{N}_{2}(1)$

29. $\mathrm{CO}(1)+\mathrm{O}_{2}(0)=\mathrm{CO}(0)+\mathrm{O}_{2}(1)$

30. $\mathrm{CO}_{2}\left(01^{1} 1\right)+\mathrm{CO}(0)=\mathrm{CO}_{2}\left(01^{1} 0\right)+\mathrm{CO}(1)$

31. $\mathrm{H}_{2}(1)+\mathrm{H}_{2} \mathrm{O}(000)=\mathrm{H}_{2}(0)+\mathrm{H}_{2} \mathrm{O}(001)$

32. $\mathrm{H}_{2}(1)+\mathrm{H}_{2} \mathrm{O}(000)=\mathrm{H}_{2}(0)+\mathrm{H}_{2} \mathrm{O}(100)$

33. $\mathrm{H}_{2}(1)+\mathrm{OH}(0)=\mathrm{H}_{2}(0)+\mathrm{OH}(1)$

34. $\mathrm{H}_{2} \mathrm{O}(001)+\mathrm{OH}(0)=\mathrm{H}_{2} \mathrm{O}(000)+\mathrm{OH}(1)$

35. $\mathrm{H}_{2} \mathrm{O}(100)+\mathrm{OH}(0)=\mathrm{H}_{2} \mathrm{O}(000)+\mathrm{OH}(1)$ 
Здесь цифры в скобках означают квантовый уровень колебательного возбуждения. Для трехатомных молекул первая цифра относится к симметричной моде $\left(v_{1}\right)$, вторая - к деформационной $\left(v_{2}\right)$, третья - к асимметричной $\left(v_{3}\right)$.

Процесс определения скоростей этих энергетических переходов и, соответственно, обменного члена $\dot{\mathrm{E}}_{V, m}$, подробно описаны в предыдущей работе автора [6] и здесь не приводятся.

\section{6. Векторная форма основной системы квазигазодинамических уравнений}

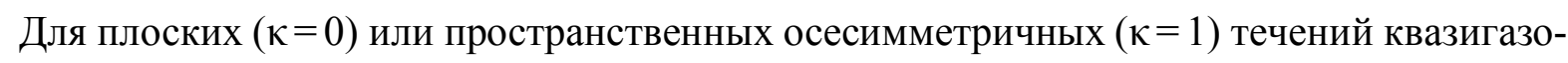
динамическая система (11), (3), (28), (23) и (8) имеет следующий вид:

$$
\begin{aligned}
& \frac{\partial \rho}{\partial t}+\frac{\partial}{\partial x}(\rho u)+\frac{1}{y^{\kappa}} \frac{\partial}{\partial y}\left(y^{\kappa} \rho v\right)=\frac{\partial g_{x}}{\partial x}+\frac{1}{y^{\kappa}} \frac{\partial}{\partial y}\left(y^{\kappa} g_{y}\right) \\
& \frac{\partial}{\partial t}(\rho u)+\frac{\partial}{\partial x}\left(\rho u^{2}+p\right)+\frac{1}{y^{\kappa}} \frac{\partial}{\partial y}\left(y^{\kappa} \rho v u\right)=\frac{\partial \Pi_{x x}}{\partial x}+\frac{1}{y^{\kappa}} \frac{\partial}{\partial y}\left(y^{\kappa} \Pi_{y x}\right) \\
& \frac{\partial}{\partial t}(\rho v)+\frac{\partial}{\partial x}(\rho u v)+\frac{\partial}{\partial y}\left(\rho v^{2}+p\right)+\kappa \frac{\rho v^{2}}{y}=\frac{\partial \Pi_{x y}}{\partial x}+\frac{1}{y^{\kappa}} \frac{\partial}{\partial y}\left(y^{\kappa} \Pi_{y y}\right)-\kappa \frac{\Pi_{\phi \phi}}{y} \\
& \frac{\partial}{\partial t}(\rho E)+\frac{\partial}{\partial x}(\rho u H)+\frac{1}{y^{\kappa}} \frac{\partial}{\partial y}\left(y^{\kappa} \rho v H\right)=\frac{\partial F_{E x}}{\partial x}+\frac{1}{y^{\kappa}} \frac{\partial}{\partial y}\left(y^{\kappa} F_{E y}\right) \\
& \frac{\partial}{\partial t}\left(\mathrm{E}_{V, m}\right)+\frac{\partial}{\partial x}\left(u \mathrm{E}_{V, m}\right)+\frac{1}{y^{\kappa}} \frac{\partial}{\partial y}\left(y^{\kappa} v \mathrm{E}_{V, m}\right)=\frac{\partial F_{m, x}}{\partial x}+\frac{1}{y^{\kappa}} \frac{\partial}{\partial y}\left(y^{\kappa} F_{m, y}\right)+\dot{\mathrm{E}}_{V, m}, \quad m=1,2, \ldots, N_{M} \\
& \frac{\partial}{\partial t}\left(\rho C_{s}\right)+\frac{\partial}{\partial x_{i}}\left(\rho C_{s} u_{i}\right)=\frac{\partial g_{s, i}}{\partial x_{i}}+\dot{w}_{s}, \quad s=1,2, \ldots, N_{C}-1,
\end{aligned}
$$

где потоки массы, импульса и энергий определятся по формулам

$$
\begin{aligned}
& g_{x}=\tau\left[\frac{\partial}{\partial x}\left(\rho u^{2}+p\right)+\frac{1}{y^{\kappa}} \frac{\partial}{\partial y}\left(y^{\kappa} \rho u v\right)\right], g_{y}=\tau\left[\frac{\partial}{\partial x}(\rho u v)+\frac{1}{y^{\kappa}} \frac{\partial}{\partial y}\left(y^{\kappa} \rho v^{2}\right)+\frac{\partial p}{\partial y}\right] ; \\
& \Pi_{x x}=\tau\left[\frac{\partial}{\partial x}\left(\rho u^{3}\right)+\frac{1}{y^{\kappa}} \frac{\partial}{\partial y}\left(y^{\kappa} \rho u^{2} v\right)+3 \frac{\partial}{\partial x}(u p)+\frac{1}{y^{\kappa}} \frac{\partial}{\partial y}\left(y^{\kappa} v p\right)\right], \\
& \Pi_{y x}=\tau\left[\frac{\partial}{\partial x}\left(\rho u^{2} v\right)+\frac{1}{y^{\kappa}} \frac{\partial}{\partial y}\left(y^{\kappa} \rho u v^{2}\right)+\frac{\partial}{\partial x}(v p)+\frac{\partial}{\partial y}(u p)\right], \\
& \Pi_{y y}=\tau\left[\frac{\partial}{\partial x}\left(\rho u v^{2}\right)+\frac{1}{y^{\kappa}} \frac{\partial}{\partial y}\left(y^{\kappa} \rho v^{3}\right)+\frac{1}{y^{\kappa}} \frac{\partial}{\partial y}\left(y^{\kappa} v p\right)+\frac{\partial}{\partial x}(u p)+2 \frac{\partial}{\partial y}(v p)\right], \\
& \Pi_{\phi \phi}=\tau\left[\frac{1}{y^{\kappa}} \frac{\partial}{\partial y}\left(y^{\kappa} v p\right)+\frac{\partial}{\partial x}(u p)+2 \frac{v p}{y}\right] ; \\
& F_{E x}=\tau\left\{\frac{\partial}{\partial x}\left[\rho u^{2}\left(H+\frac{p}{\rho}\right)\right]+\frac{1}{y^{\kappa}} \frac{\partial}{\partial y}\left[y^{\kappa} \rho u v\left(H+\frac{p}{\rho}\right)\right]+\frac{\partial}{\partial x}\left[p\left(\frac{u^{2}}{2}+\frac{v^{2}}{2}\right)\right]+h \frac{\partial p}{\partial x}+\frac{1}{\operatorname{Pr}} p \frac{\partial h}{\partial x}\right\}, \\
& F_{E y}=\tau\left\{\frac{\partial}{\partial x}\left[\rho u v\left(H+\frac{p}{\rho}\right)\right]+\frac{1}{y^{\kappa}} \frac{\partial}{\partial y}\left[y^{\kappa} \rho v^{2}\left(H+\frac{p}{\rho}\right)\right]+\frac{\partial}{\partial y}\left[p\left(\frac{v^{2}}{2}+\frac{u^{2}}{2}\right)\right]+h \frac{\partial p}{\partial y}+\frac{1}{\operatorname{Pr}} p \frac{\partial h}{\partial y}\right\} ;
\end{aligned}
$$




$$
\begin{aligned}
& F_{V, x}=\frac{\mathrm{E}_{V, m}}{\rho} g_{x}+\tau\left[\left(\rho u^{2}+\frac{1}{\operatorname{Pr}} p\right) \frac{\partial}{\partial x}\left(\frac{\mathrm{E}_{V, m}}{\rho}\right)+(\rho u v) \frac{\partial}{\partial y}\left(\frac{\mathrm{E}_{V, m}}{\rho}\right)\right], \\
& F_{V, y}=\frac{\mathrm{E}_{V, m}}{\rho} g_{y}+\tau\left[(\rho u v) \frac{\partial}{\partial x}\left(\frac{\mathrm{E}_{V, m}}{\rho}\right)+\left(\rho v^{2}+\frac{1}{\operatorname{Pr}} p\right) \frac{\partial}{\partial y}\left(\frac{\mathrm{E}_{V, m}}{\rho}\right)\right] ; \\
& g_{s, x}=C_{s} g_{x}+\tau\left[\left(\rho u^{2}+\frac{1}{\mathrm{Sc}} p\right) \frac{\partial C_{s}}{\partial x}+\rho u v \frac{\partial C_{s}}{\partial y}\right], \\
& g_{s, y}=C_{s} g_{y}+\tau\left[(\rho u v) \frac{\partial C_{s}}{\partial x}+\left(\rho v^{2}+\frac{1}{\mathrm{Sc}} p\right) \frac{\partial C_{s}}{\partial y}\right]
\end{aligned}
$$

Систему (29) удобно представить в векторной форме

$$
\frac{\partial \mathbf{U}}{\partial t}+\frac{\partial \mathbf{F}_{I}}{\partial x}+\frac{1}{y^{\kappa}} \frac{\partial}{\partial y}\left(y^{\kappa} \mathbf{G}_{I}\right)+\kappa \frac{1}{y} \mathbf{H}_{I}=\frac{\partial \mathbf{F}_{V}}{\partial x}+\frac{1}{y^{\kappa}} \frac{\partial}{\partial y}\left(y^{\kappa} \mathbf{G}_{V}\right)+\kappa \frac{1}{y} \mathbf{H}_{V}+\mathbf{S}
$$

где

$$
\mathbf{U}=\left(\rho, \rho u, \rho v, \rho E, \mathrm{E}_{V, 1}, \ldots, \mathrm{E}_{V, N m}, \rho C_{1}, \ldots, \rho C_{N c-1}\right)^{T},
$$

$$
\begin{gathered}
\mathbf{F}_{I}=\left(\begin{array}{c}
\rho u \\
\rho u^{2}+p \\
\rho u v \\
\rho u H \\
u \mathrm{E}_{V, m} \\
\rho u C_{s}
\end{array}\right), \mathbf{G}_{I}=\left(\begin{array}{c}
\rho v \\
\rho u v \\
\rho v^{2}+p \\
\rho v H \\
v \mathrm{E}_{V, m} \\
\rho v C_{s}
\end{array}\right), \mathbf{H}_{I}=\left(\begin{array}{c}
0 \\
0 \\
-p \\
0 \\
0 \\
0
\end{array}\right), \mathbf{F}_{V}=\left(\begin{array}{c}
g_{x} \\
\Pi_{x x} \\
\Pi_{x y} \\
F_{E x} \\
F_{m, x} \\
g_{s, x}
\end{array}\right), \mathbf{G}_{V}=\left(\begin{array}{c}
g_{y} \\
\Pi_{y x} \\
\Pi_{y y} \\
F_{E y} \\
F_{m, y} \\
g_{s, y}
\end{array}\right), \mathbf{H}_{V}=\left(\begin{array}{c}
0 \\
0 \\
-\Pi_{\phi \phi} \\
0 \\
0 \\
0
\end{array}\right) \\
\mathbf{S}=\left(0,0,0,0, \dot{\mathrm{E}}_{V, 1}, \ldots, \dot{\mathrm{E}}_{V, N m}, \dot{w}_{1}, \ldots, \dot{w}_{N c-1}\right)^{T}
\end{gathered}
$$

\section{7. Сравнение с экспериментом и результатами расчета других авторов}

C точки зрения численного решения система уравнений (30) мало чем отличается от системы уравнений Навье-Стокса, описывающей течение плотного газа, поэтому для численного решения основных квазигазодинамических уравнений использовался метод, описанный в работе [7], и программа Universe CFD, разработанная в МАИ на кафедре Авиационнокосмической теплотехники. Для эффективного решения "жестких" уравнений переноса колебательных энергий использовалась методика, описанная в работе [8].

Для проверки методики, основанной на решении КГД уравнений, проведено сравнение результатов расчета толщины скачка уплотнения в одномерном течении с экспериментальными данными, представленными в работе [9]; с результатами расчета с использованием уравнений Навье - Стокса, а также метода Монте-Карло [10].

В [9] в качестве рабочего тела использовался Ar. Толщина скачка $t_{\rho}$ определялась по профилю плотности (по максимальному градиенту).

На рис. 1 представлено отношение длины свободного пробега молекулы 1 к толщине скачка уплотнения в зависимости от числа Маха набегающего потока. Очевидно, что расчет с использованием КГД уравнений удовлетворительно согласуется с экспериментом и с расчетом по методу Монте-Карло. При применения уравнений Навье-Стокса толщина скачка уплотнения получается почти в 2 раза меньше, чем в эксперименте. 


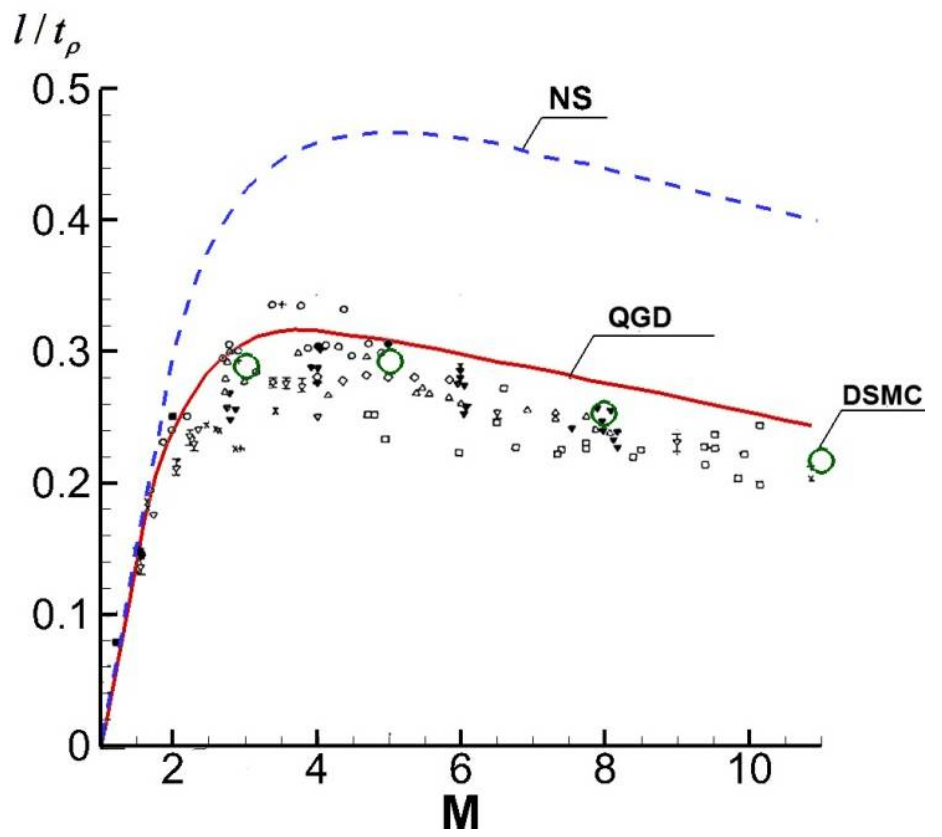

Рис.1. Отношение длины свободного пробега молекулы к толщине скачка уплотнения в зависимости от числа Маха набегающего потока:

NS - расчет с использованием уравнений Навье-Стокса; DSMC - расчет с использованием метода Монте-Карло; QGD - расчет с использованием квазигазодинамических уравнений.

Мелкими значками представлены экспериментальные данные из [9].

Аналогичные результаты получены в работе [4].

\section{8. Результаты расчета струи двигателя на высотах от 100 до 200 км}

Для анализа предложенной методики рассматривалась струя модельного жидкостного двигателя с параметрами из работы [11] (см. Табл.1).

Таблица 1

\section{Параметры на срезе сопла}

\begin{tabular}{|c|c|c|c|c|c|c|c|c|}
\hline$T_{a}, \mathrm{~K}$ & $U_{a}, \mathrm{M} / \mathrm{c}$ & $R_{a}, \mathrm{M}$ & $p_{a}$, атм & $\mathrm{H}_{2}$ & $\mathrm{H}_{2} \mathrm{O}$ & $\mathrm{CO}$ & $\mathrm{CO}_{2}$ & $\mathrm{~N}_{2}$ \\
\hline 2000 & 2500 & 0.5 & 0.3 & 0.05 & 0.4 & 0.05 & 0.15 & 0.35 \\
\hline
\end{tabular}

Химический состав задан в мольных долях.

Рассматривалось 7 высот полета: 100км (скорость внешнего потока $2950 \mathrm{~m} / \mathrm{c}), 110 \kappa \mathrm{k}$ $(3130 \mathrm{~m} / \mathrm{c}), 120$ км $(3340 \mathrm{M} / \mathrm{c}), 130$ км $(3550 \mathrm{~m} / \mathrm{c}), 150$ км (4060 м/с), 180км (4900 м/с), 200км (5500 $\mathrm{M} / \mathrm{c})$.

На каждой высоте проводилось 4 варианта расчета: 1) решение системы уравнений Навье-Стокса с учетом термической неравновесности (на графиках обозначен как "NS"' или "NS Neq"); 2) решение системы квазигазодинамических уравнений с учетом термической неравновесности (на графиках обозначен как "QGD"' или "QGD Neq"); 3) решение системы уравнений Навье-Стокса в предположении термического равновесия, т.е. $T=T_{T R}=T_{V, m}$; 4) решение системы квазигазодинамических уравнений в предположении термического равновесия (на графиках обозначен как "QGD Eq").

На основе этого получены пространственные распределения газодинамических параметров течения для всех указанных вариантов. Эти распределения затем использовались для расчета излучения струй в диапазоне длин волн от 1 до 7 мкм. 
Физико-математическая модель расчета оптических характеристик газовых потоков с учетом колебательной неравновесности подробно описана в предыдущих работах $[6,12]$.

В них разработана методика расчета теплового излучения колебательно неравновесного газа на основе метода $k$-распределения, получены формулы для расчета средних по полосе пропускательной способности и эффективной функции Планка для колебательно неравновесной газовой смеси, создана база данных для каждого компонента, в которой зависимость коэффициента поглощения получена для различных значений волновых чисел, температур (всех энергетических мод) и парциальных давлений газовых компонентов.

Наиболее интересными являются следующие диапазоны длин волн: 1) в районе 2.7 мкм излучение $\mathrm{H}_{2} \mathrm{O}$ с переходами с верхнего уровня третьей моды $\left.v_{3} ; 2\right)$ колебательная температура $T_{\mathrm{H} 2 \mathrm{O}}\left(v_{2}\right)$ наиболее важна в районе 6 мкм; 3$)$ в районе 2.7 мкм $\left(3400 \div 3800 \mathrm{~cm}^{-1}\right)$ излучение $\mathrm{CO}_{2}$ связано с переходами с верхних уровней $\left.2 v_{2}+v_{3}, v_{1}+v_{3} ; 4\right)$ очень сильная линия излучения $\mathrm{CO}_{2}$ находится в районе 4.3 мкм и связана с переходами с верхнего уровня третьей моды $\left.v_{3} ; 5\right)$ наконец, линия излучения СО находится в районе 4.7 мкм.

Поскольку $\mathrm{CO}_{2}$ и $\mathrm{H}_{2} \mathrm{O}$ практически всегда присутствуют в рассматриваемых объектах, особое внимание уделено диапазону $2.7 \div 2.9$ мкм.

На рис. 2 представлена зависимость интегральной интенсивности излучения (Bт/cp) от высоты в диапазоне длин волн $1 \div 7$ мкм. Здесь и далее рассматривалась длина струи 5 км. Расчет проведен с учетом термической неравновесности. Начиная со 120 км (длина свободного пробега молекулы в окружающей атмосфере на этой высоте составляет $~ 3$ м) в расчете с использованием НС наблюдается существенный рост излучения с высотой. В расчете с использованием КГД интегральное излучение практически не меняется по высоте и отличается от предыдущего расчета в 4 раза на 200 км.

Аналогичная картина наблюдается и для интегрального излучения в диапазоне $2.7 \div 2.9$ мкм, представленного на рис. 3.

Высотное распределение излучения, полученное с использованием КГД, более соответствует наблюдаемой в реальности картине.

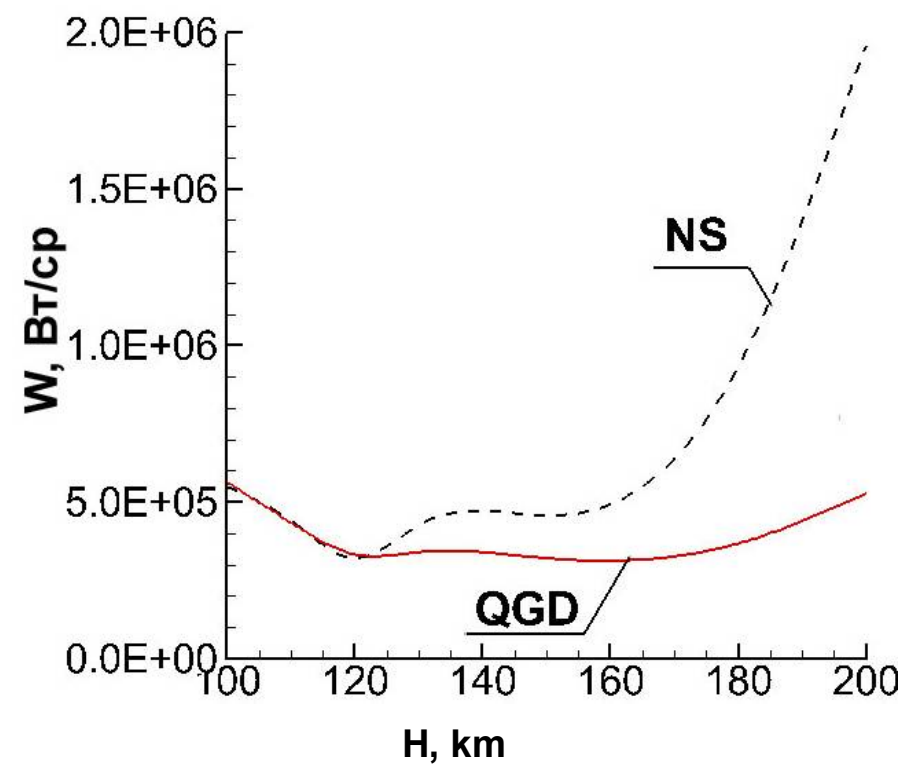

Рис. 2. Зависимость интегральной интенсивности излучения (диапазон $1 \div 7$ мкм) от высоты: $\mathrm{NS}$ - расчет с использованием уравнений Навье-Стокса с учетом термической неравновесности; QGD - расчет с использованием квазигазодинамических уравнений с учетом термической неравновесности 


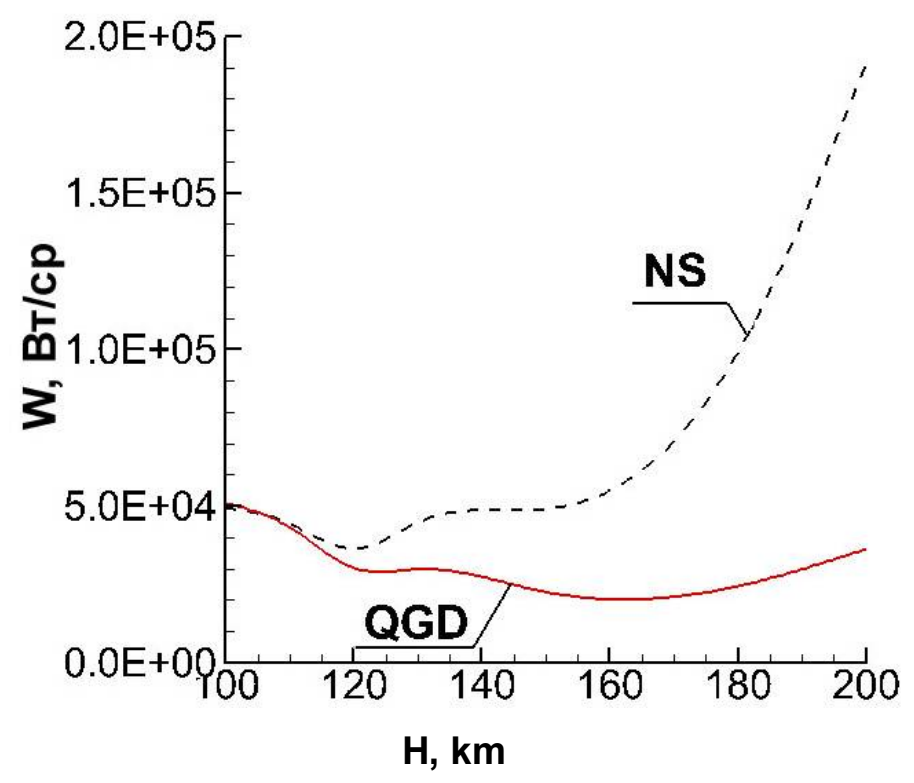

Рис. 3. Зависимость интегральной интенсивности излучения (диапазон $2.7 \div 2.9$ мкм) от высоты: NS - расчет с использованием уравнений Навье-Стокса с учетом термической неравновесности; QGD - расчет с использованием квазигазодинамических уравнений с учетом термической неравновесности

Более подробно полученные результаты показаны на рис. 4, 5, 6, на которых представлено распределение погонной интенсивности излучения (Вт/(cp·cм) ) по длине струи для трех высот: 100 км, 150 км и 180 км.

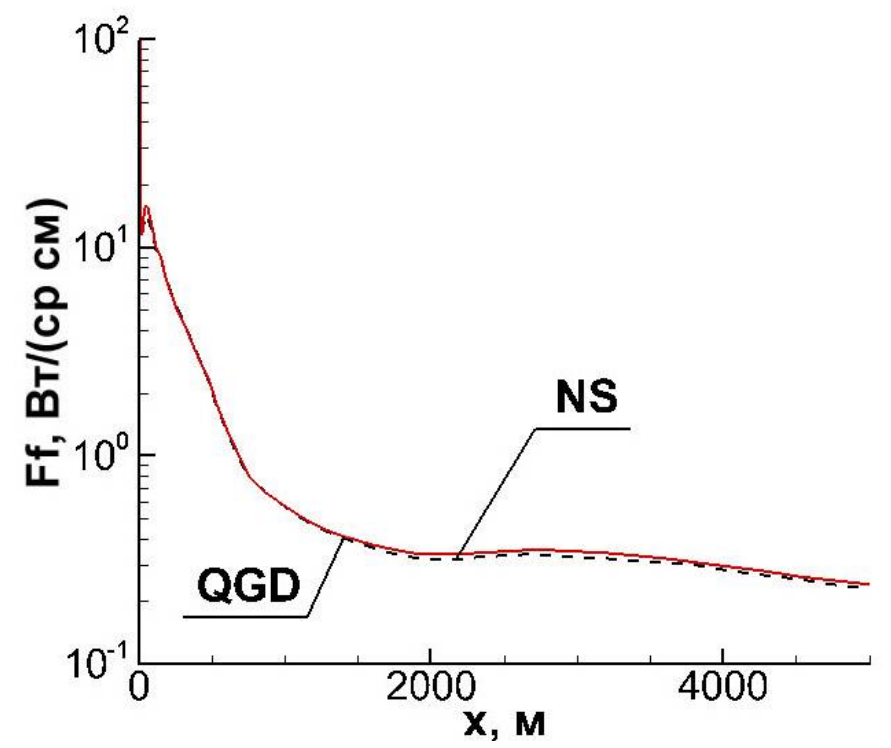

Рис. 4. Распределение погонной интенсивности излучения по длине струи для высоты 100 км в диапазоне длин волн $1 \div 7$ мкм. Пояснения см. рис. 2

На высоте 100 км оба варианта расчета практически совпадают.

На высотах 150, 180 км наблюдается существенное различие. Наибольшее отличие погонной интенсивности излучения в обоих диапазонах проявляется на расстоянии $400 \div 1000$ м от среза сопла. 


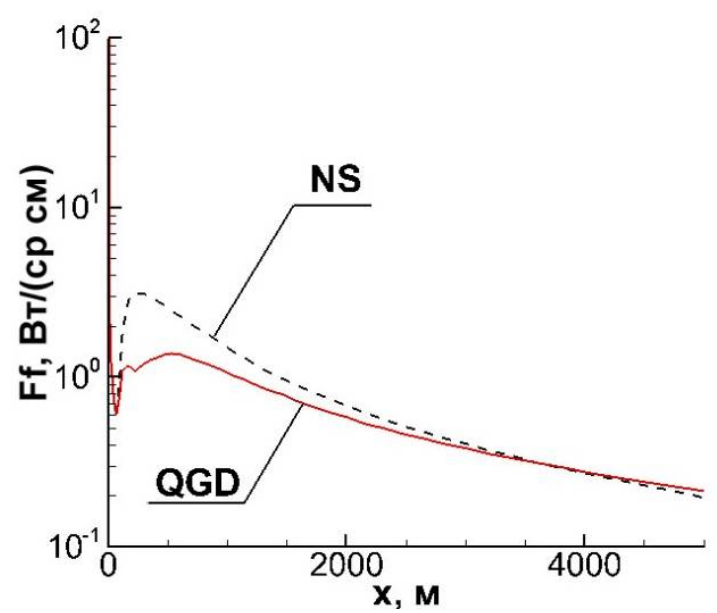

(a)

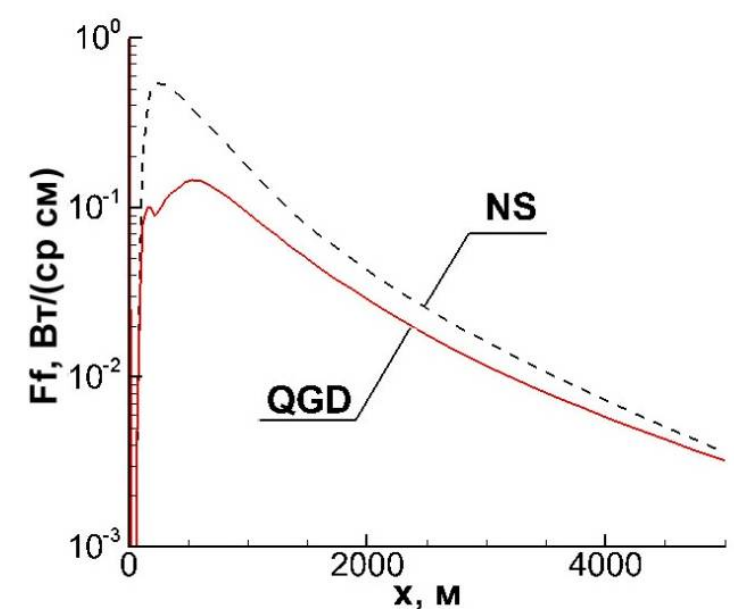

(b)

Рис. 5. Распределение погонной интенсивности излучения по длине струи для высоты 150 км: a) в диапазоне $1 \div 7$ мкм; b) в диапазоне $2.7 \div 2.9$ мкм. Пояснения см. рис. 2

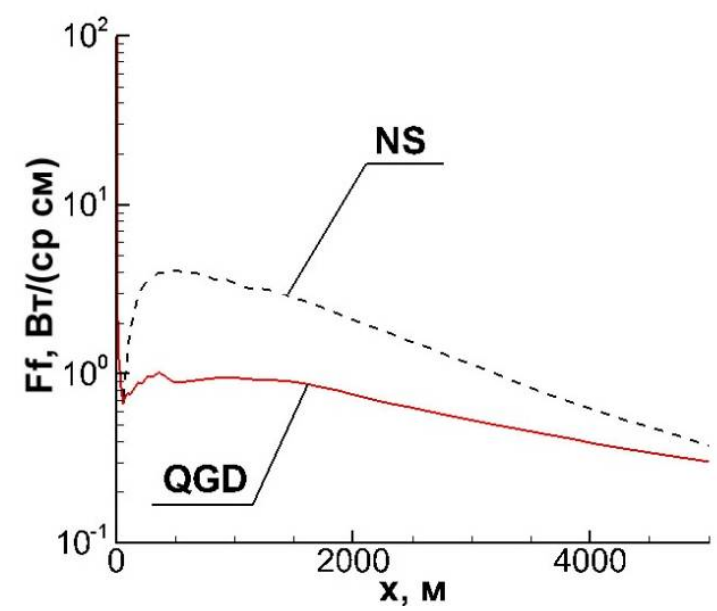

(a)

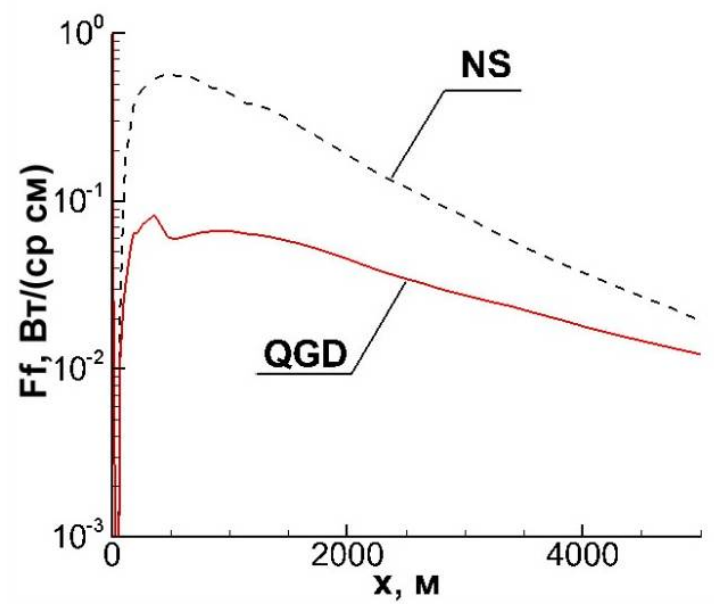

(b)

Рис. 6. Распределение погонной интенсивности излучения по длине струи для высоты 180 км: a) в диапазоне $1 \div 7$ мкм; b) в диапазоне $2.7 \div 2.9$ мкм. Пояснения см. рис. 2

Рассмотрим поперечные профили температур на расстоянии 400 м от среза сопла.

На рис. 7 представлены поперечные профили температур (поступательно-вращательной и колебательных) на расстоянии 400 м от среза сопла на высоте 100км.

Расчеты по НС и КГД мало отличаются.

Интересно проанализировать спектральную интенсивность излучения для обоих случаев. Она показана на рис. 8. Спектры практически совпадают.

Мы видим сильные линии $\mathrm{H}_{2} \mathrm{O}$ (районы 2.7 мкм и 6 мкм), $\mathrm{CO}_{2}$ (районы 2.7 мкм и 4.3 мкм) и СО (район 4.7 мкм). Действительно, соответствующие колебательные температуры, связанные с излучением в этих диапазонах спектра, еще достаточно велики на этой высоте.

На рис. 9 представлены поперечные профили температур (поступательно-вращательной и колебательных) на расстоянии 400 м от среза сопла на высоте 150 км. Расчеты по НС и КГД уже заметно отличаются. Это относится как к поступательно-вращательной температуре, так и колебательным температурам. Колебательные температуры $T_{\mathrm{CO}_{2}}\left(v_{3}\right)$ и $T_{\mathrm{CO}}$ практически заморожены в обоих случаях и их вклад в спектральное излучение (рис. 10) одинаков соответственно в районах 4.3 мкм и 4.7 мкм. 


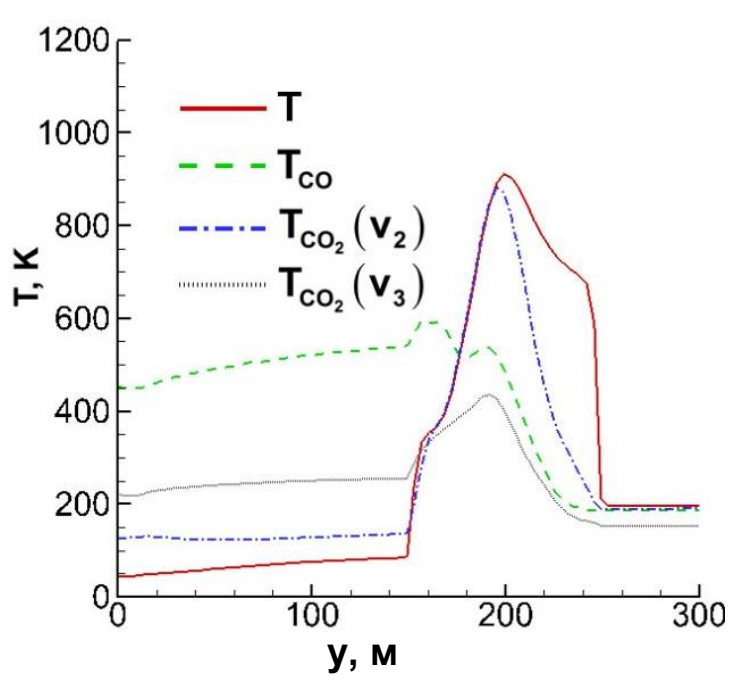

(a)

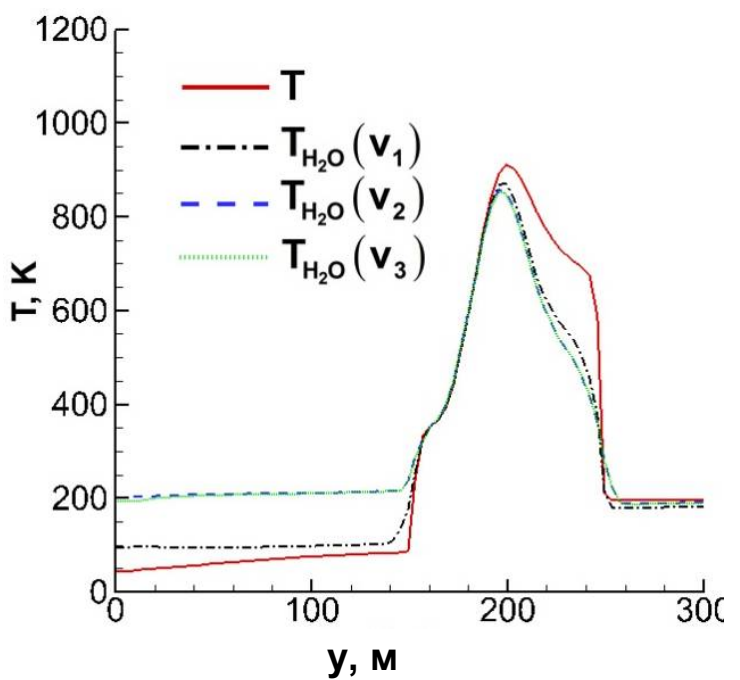

(c)

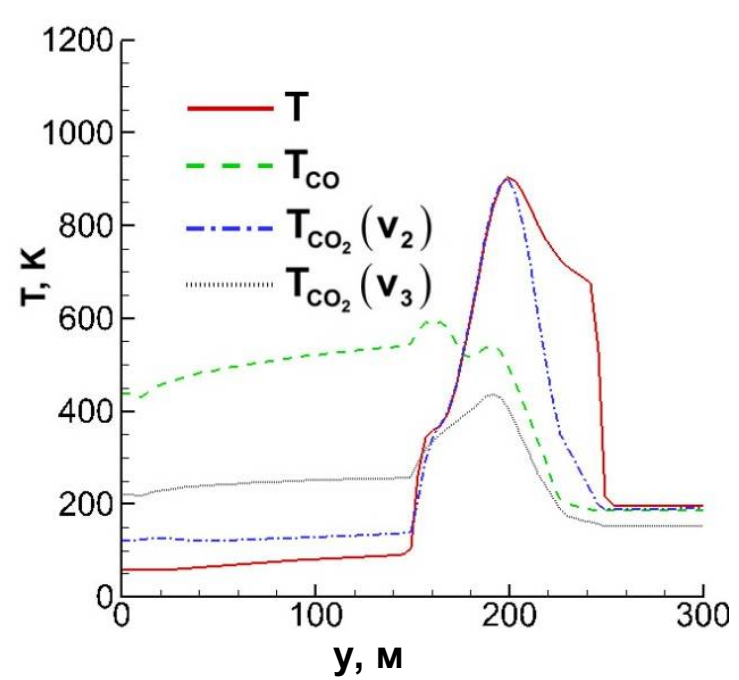

(b)

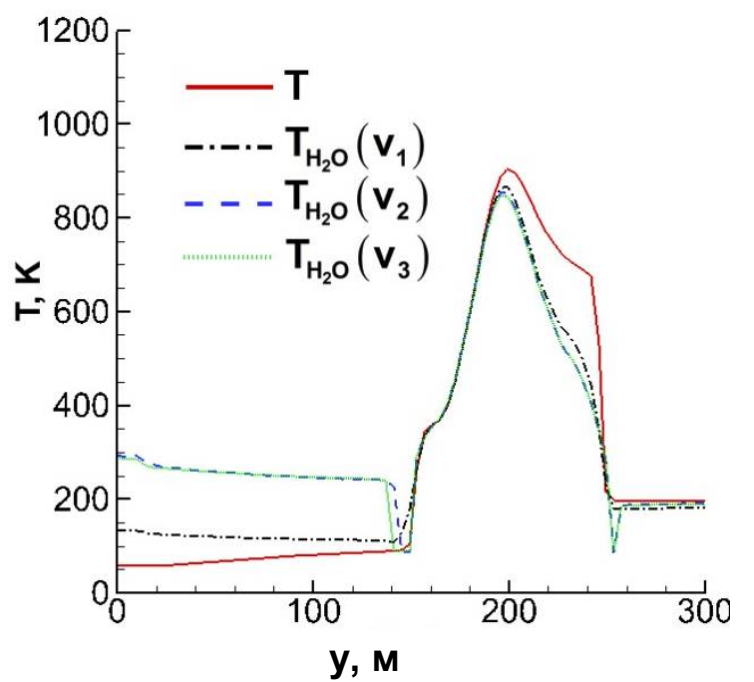

(d)

Рис. 7. $h=100$ км. Поперечные профили температур на расстоянии 400 м от среза сопла на высоте 100км.

(a), (c) - расчет с использованием уравнений Навье-Стокса;

(b), (d) - расчет с использованием квазигазодинамических уравнений

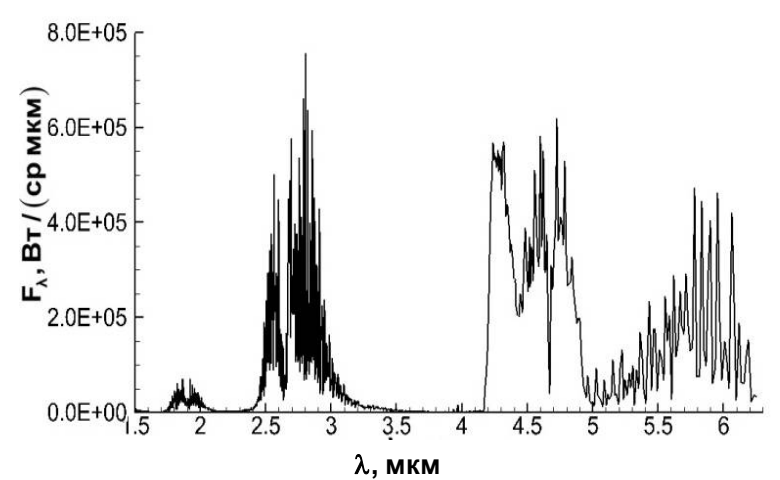

(a)

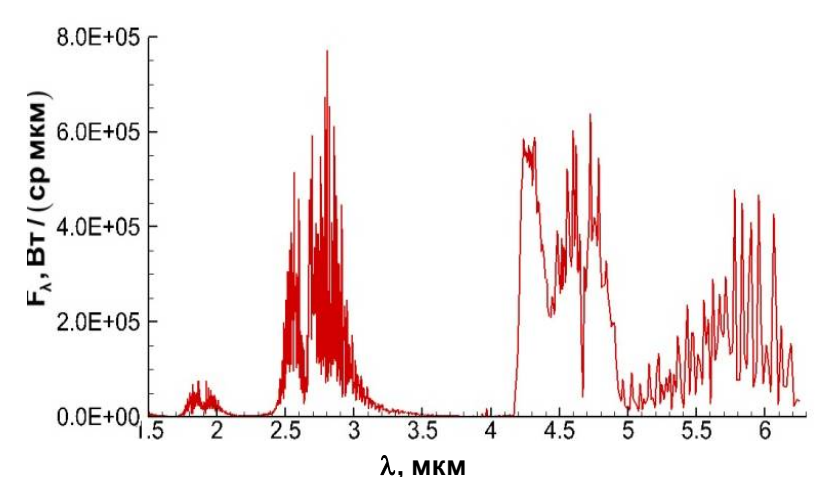

(b)

Рис. 8 . $h=100$ км. Спектральная интенсивность излучения на высоте 100 км.

(a) - расчет с использованием уравнений Навье-Стокса с учетом термической неравновесности; (b) - расчет с использованием квазигазодинамических уравнений с учетом термической неравновесности. 


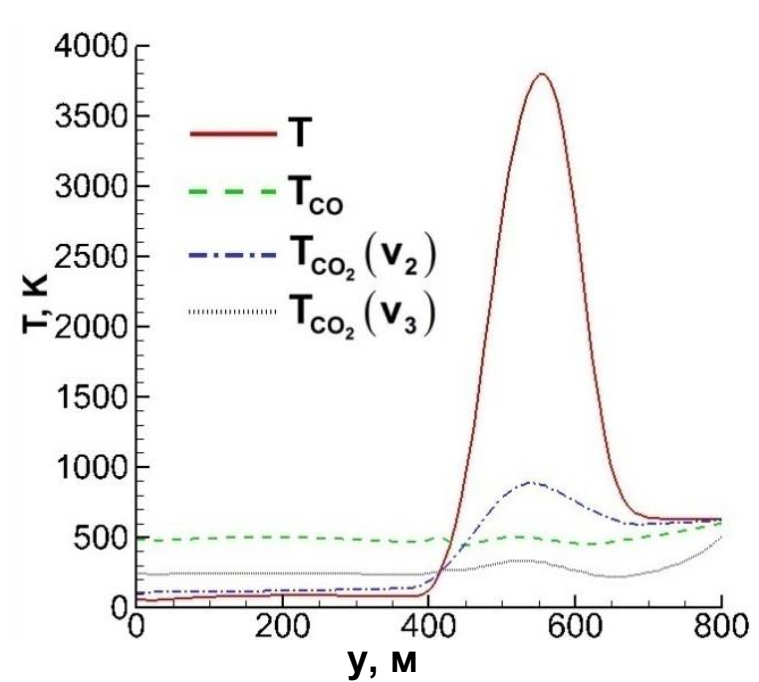

(a)

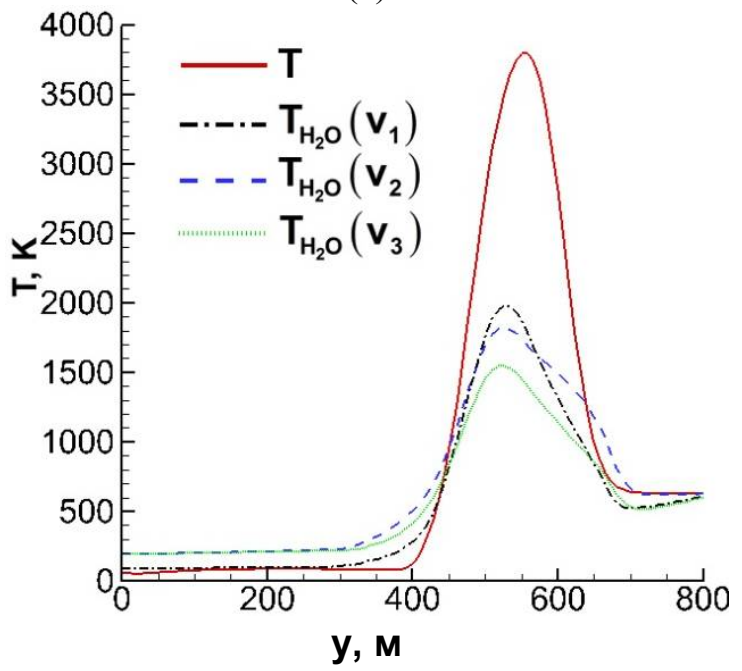

(c)

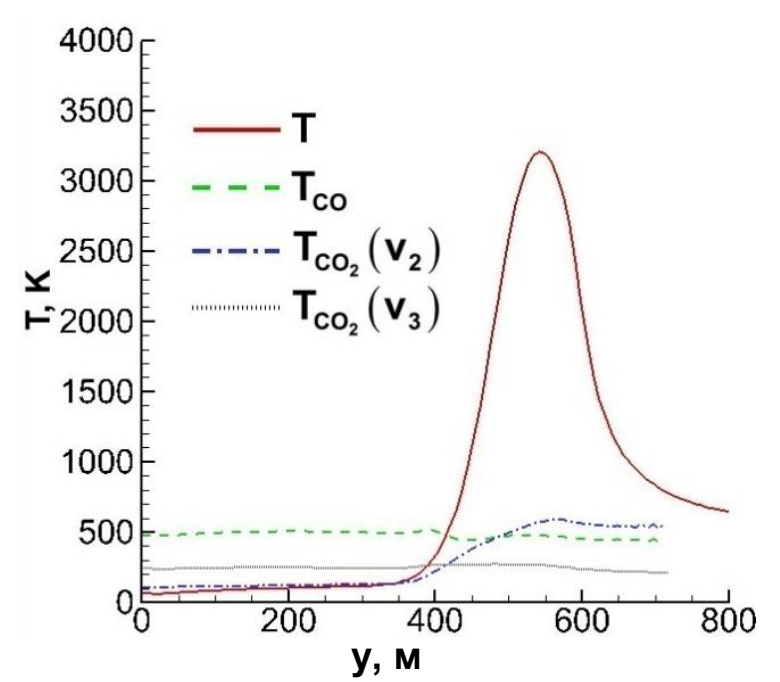

(b)

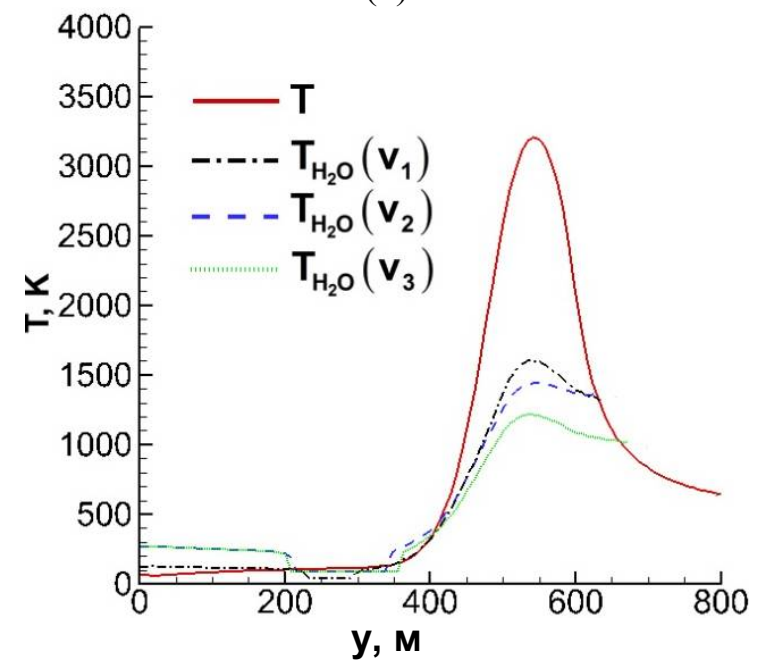

(d)

Рис. 9. $h=150$ км. Поперечные профили температур на расстоянии 400 м от среза сопла на высоте 150 км. Пояснения см. рис. 7

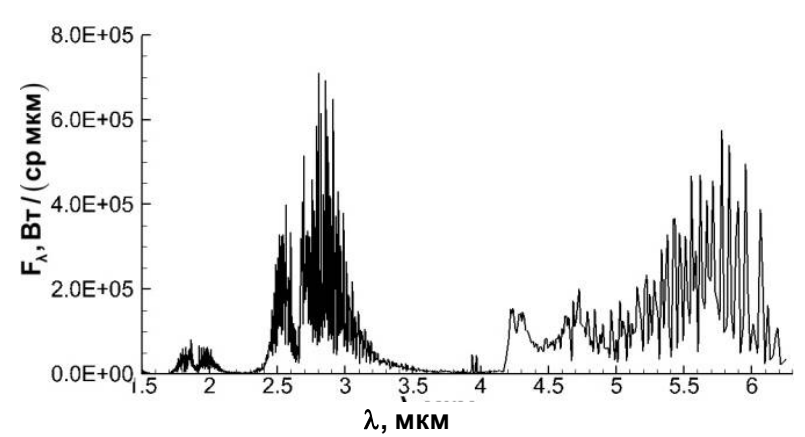

(a)

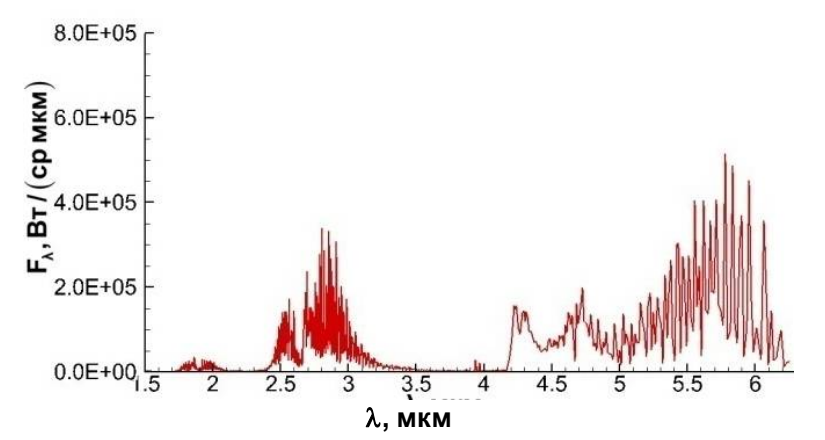

(b)

Рис. 10. $h=150$ км. Спектральная интенсивность излучения на высоте 150 км. Пояснения см. рис. 8

Влияние разреженности гораздо сильнее проявляется для колебательных температур воды и $T_{\mathrm{CO}_{2}}\left(v_{2}\right)$ и, соответственно, в диапазоне спектра 2.7 мкм.

Аналогичные результаты получены для высоты 180 км (рис. 11 и 12). Влияние разреженности газа проявляется еще сильнее: все температуры значительно ниже, а все важные линии спектра существенно ослабевают. 


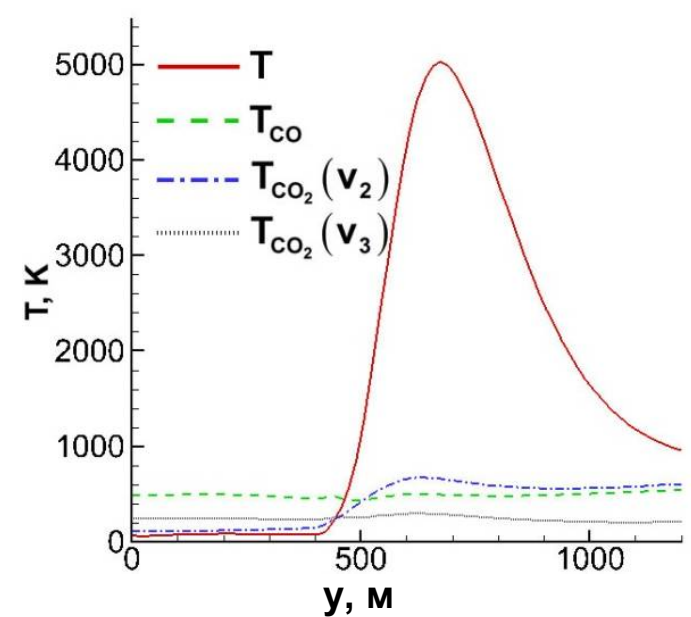

(a)

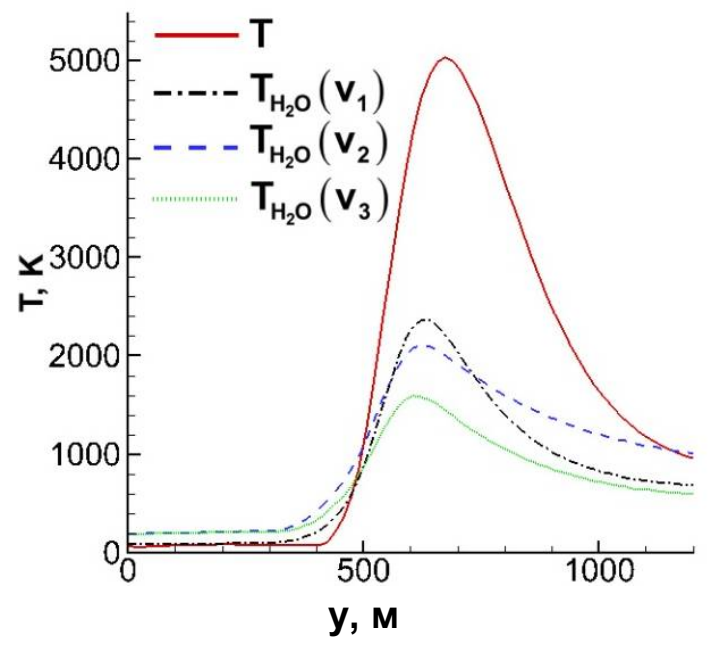

(c)

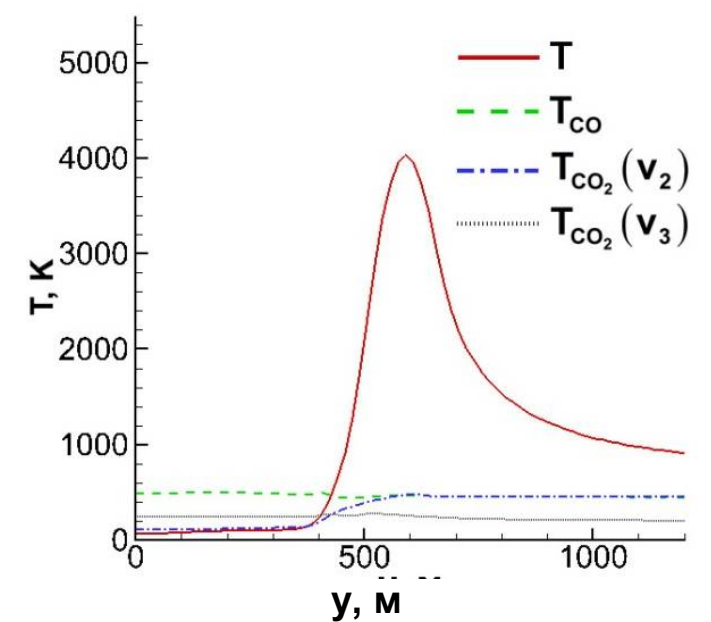

(b)

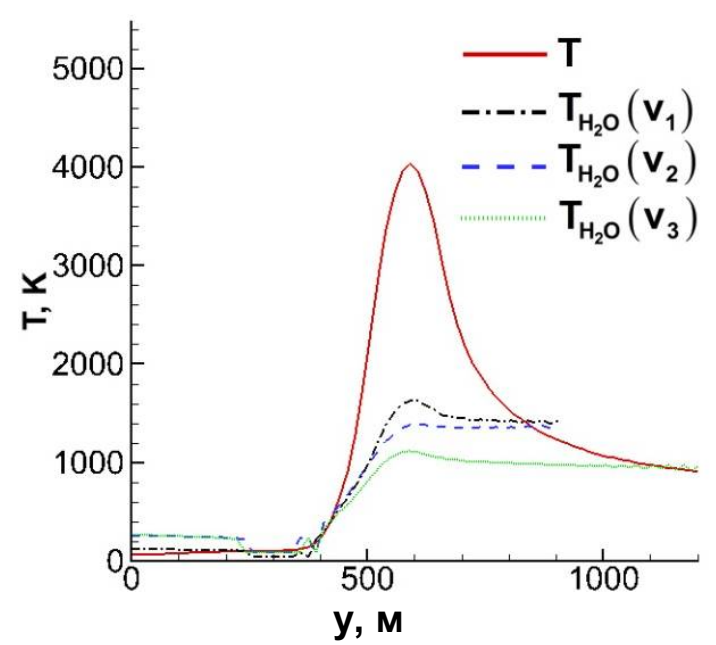

(d)

Рис. 11. $h=180$ км. Поперечные профили температур на расстоянии 400 м от среза сопла на высоте 180 км. Пояснения см. рис. 7

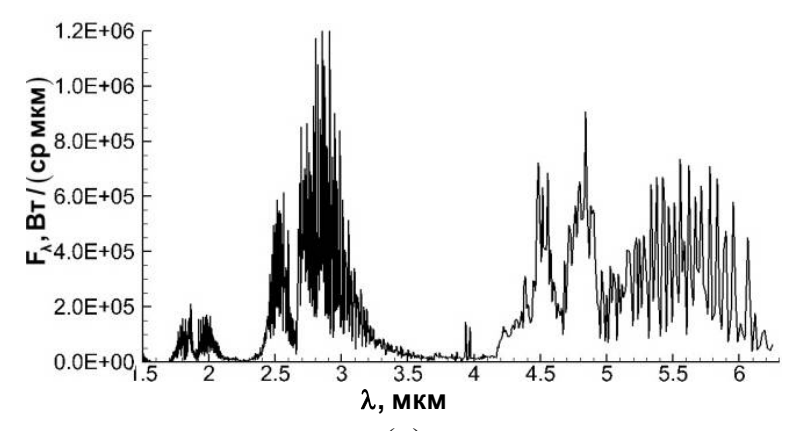

(a)

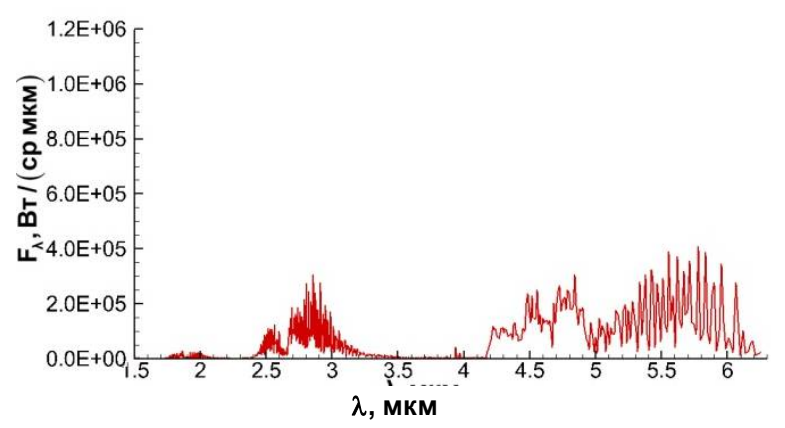

(b)

Рис. 12. $h=180$ км. Спектральная интенсивность излучения на высоте 180 км. Пояснения см. рис. 8

В качестве примера пространственных распределений параметров, на рис. 13 показано распределение температуры. Видно, что учет разреженности в расчетах приводит к снижению температуры в слое смешения на протяжении всей длины струи.

В заключение, в качестве иллюстрации важности учета термической неравновесности, на рис. 14, 15 приведено сравнение результатов расчетов решение системы КГД уравнений с учетом неравновесности (QGD Neq) и без ее учета (QGD Eq). 


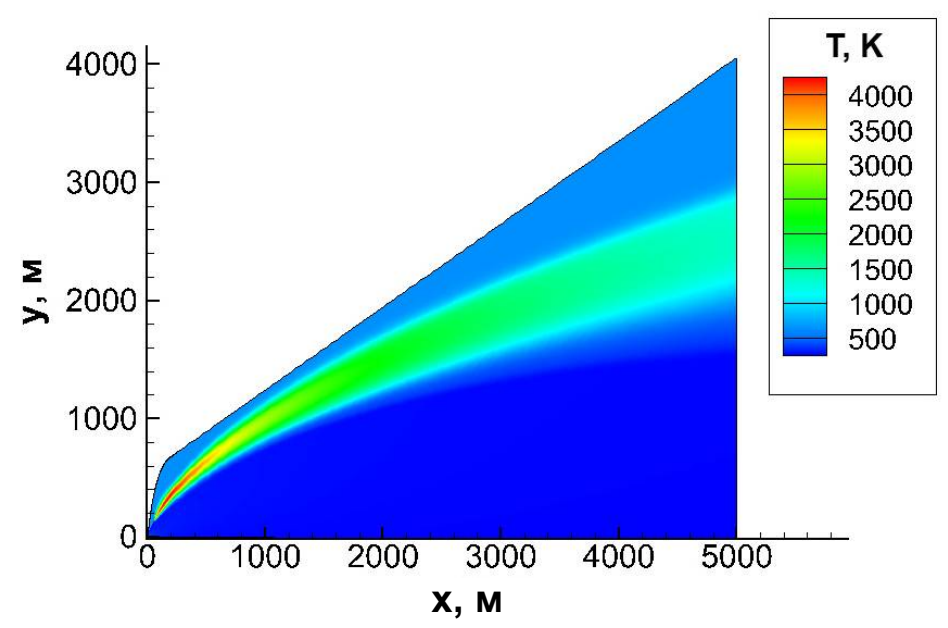

(a)

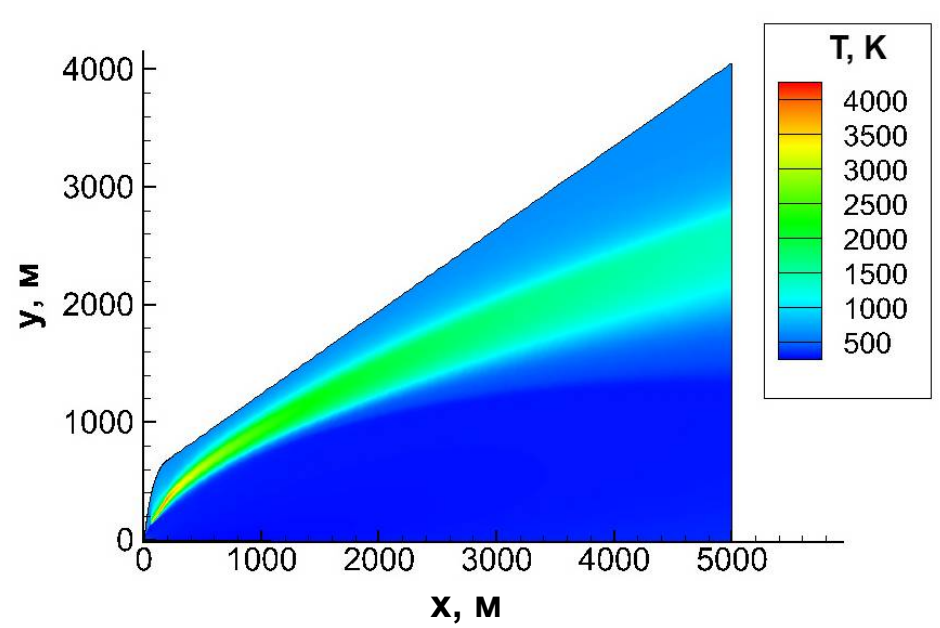

(b)

Рис. 13. $h=150$ км. Пространственное распределение поступательно-вращательной температуры на высоте 150 км.

(a) - расчет с использованием уравнения Навье-Стокса с учетом термической неравновесности; (b) - расчет с использованием квазигазодинамических уравнений с учетом термической неравновесности

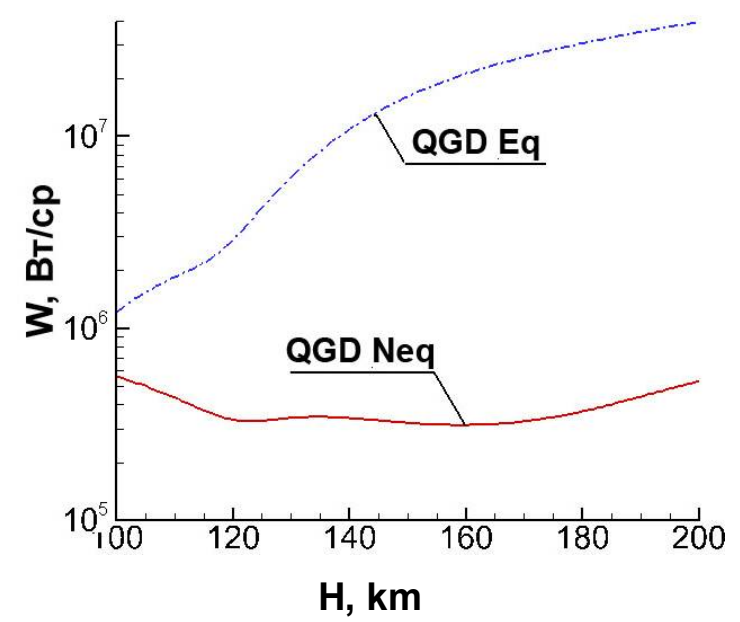

Рис. 14. Зависимость интегральной интенсивности излучения (диапазон $1 \div 7$ мкм) от высоты: QGD Eq - расчет с использованием квазигазодинамических уравнений в предположении термического равновесия; QGD Neq - расчет с использованием квазигазодинамических уравнений с учетом термической неравновесности 


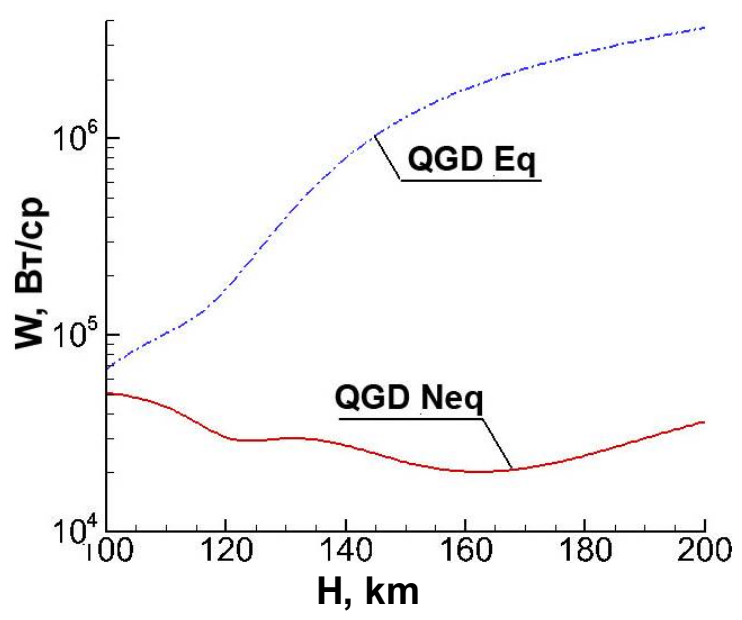

Рис. 15. Зависимость интегральной интенсивности излучения (диапазон $2.7 \div 2.9$ мкм) от высоты: QGD Eq - расчет с использованием квазигазодинамических уравнений в предположении термического равновесия; QGD Neq - расчет с использованием квазигазодинамических уравнений с учетом термической неравновесности

Видно, что отличие двух расчетов проявляется уже на высоте 100 км и на 200-х км достигает 2-х порядков.

\section{9. Заключение}

Разработана математическая модель высотных струй, основанная на квазигазодинамических (КГД) уравнениях.

Из уравнения Больцмана получены уравнения неразрывности компонентов газовой смеси и уравнения переноса колебательных энергий в квазигазодинамической постановке.

С точки зрения компьютерных ресурсов расчет с использованием КГД уравнений сопоставим с решением стандартных уравнений Навье-Стокса и существенно экономичнее методов прямого численного моделирования - методов Монте-Карло.

Сопоставление результатов расчета прямого скачка уплотнения по данной методике с экспериментальными данными и результатами расчетов других авторов показало удовлетворительное совпадение.

Проведен тестовый анализ струи, истекающей из типичного жидкостного ракетного двигателя на больших высотах, который показал, что:

1) при расчете с использованием КГД уравнений интенсивность излучения существенно ниже, чем при расчете с использованием стандартной системы уравнений Навье-Стокса;

2) это отличие начинается примерно с высоты полета 120 км, и на высоте 200 км достигает почти 5 раз;

3) интенсивность излучения при использовании КГД уравнений практически не зависит от высоты полета;

4) более низкая интенсивность излучения, полученная при использовании КГД уравнений по сравнению с НС, обусловлена тем, что температуры (поступательная, вращательные и колебательные) в слое смешения струи с окружающим воздухом имеют более низкое значение;

5) наиболее важное значение указанный эффект имеет в диапазоне длин волн в районе 2.7 мкм, т.к. использование КГД уравнений приводит к существенному уменьшению колебательных температур, соответствующих излучению в данном диапазоне; 
6) расчет с использованием предположения о термическом равновесии (т.е. равновесия между всеми энергетическими модами) приводит к совершенно нефизичным результатам: излучение завышается на 1-2 порядка.

Меньшая интенсивность излучения, получаемая при использовании КГД уравнений, по сравнению с уравнениями Навье-Стокса объясняется тем, что в модели КГД уравнений учитывается увеличение толщины скачка и уменьшается температура в сжатом слое.

\section{Литература}

1. Bird, G.A. Molecular Gas Dynamics and the Direct Simulation of Gas Flows / G.A. Bird. - Oxford, Clarendon press, 1994. $-458 \mathrm{p}$.

2. Зея Мьо Мьинт. Построение метода Монте-Карло для решения задач высотной аэродинамики / Зея Мьо Мьинт, А.Ю. Хлопков, Чжо Зин. // ТРУ ДЫ МФТИ. - 2014. - Том 6. - № 1. - С. 92-100.

3. Gimelshein, S.F., Levin D.A., and Alexeenko A.A., Modeling of Chemically Reacting Flows from a Side Jet at High Altitudes / S.F. Gimelshein, D.A. Levin., and Alexeenko A.A. // Journal of Spacecraft and Rockets. - 2004. - Vol. 41. - No. 4. - Pp. 582-591.

4. Елизарова, Т.Г. Теоретическое и численное исследование квазигазодинамических и квазигидродинамических уравнений / Т.Г. Елизарова, Ю.В. Шеретов // Журнал вычислительной математики и математической физики. - 2001. - Том 41. - № 2. - С. 239-255.

5. Елизарова, Т.Г. Квазигазодинамические уравнения и методы расчета вязких течений / Т.Г. Елизарова. - М.: Научный Мир, 2007. - 350 с.

6. Молчанов, А.М. Расчет теплового излучения колебательно неравновесного газа методом k-распределения / Физико-химическая кинетика в газовой динамике. - 2015. - Т. 16. - Вып. 1. http://chemphys.edu.ru/issues/2015-16-1/articles/317/

7. Molchanov, A.M. Numerical Simulation of Supersonic Chemically Reacting Turbulent Jets / A.M. Molchanov // AIAA Paper 2011-3211. - 2011. - 37 p.

8. Молчанов, А.М. Эффективный численный метод расчета химически неравновесных течений / А.М. Молчанов // Фундаментальные исследования. - 2018. - № 2. - С. 28-33.

9. Alsmeyer, H. Density Profiles in Argon and Nitrogen Shock Waves Measured by the Absorption of an Electron Beam / H. Alsmeyer // Journal of Fluid Mechanics. - 1976. - Vol. 74. - Pp. 497-513.

10. Weaver D. P. Comparison of Burnett, Super-Burnett, and Monte Carlo Solutions for Hypersonic Shock Structure / D. P. Weaver, D. H. Campbell, E. P. Muntz // Rarefied Gas Dynamics: Theoretical and Computational Techniques, Progress in Astronautics and Aeronautics. - 1989. - Pp. 374-395.

11. Vitkin E.I.A Physico-Mathematical Model of Rocket Exhaust Plumes / E.I. Vitkin, V.G. Karelin, A.A Kirillov, A.S. Suprun, Ju.V. Khadyka // Int. J. Heat Mass Transfer. - 1997. - Vol.40. - No 5. Pp. $1227-1241$.

12. Молчанов, А.М. Узкополосная база данных для расчета излучения продуктов сгорания с использованием k-распределения / А.М. Молчанов, П.В. Никитин // Тепловые процессы в технике. - 2014. - №10. -С. 448-455.

Статья поступила в редакцию 31 октября 2018 г. 